\title{
Existence of minimizers for non-quasiconvex functionals arising in optimal design
}

\author{
by \\ Grégoire ALLAIRE \\ Commissariat à l'Energie Atomique, DRN/DMT/SERMA, CEA Saclay \\ 91191 Gif sur Yvette, France, Laboratoire d'Analyse Numérique, Universitê Paris 6 \\ and
}

\section{Gilles FRANCFORT}

Institut Galilée, Université Paris-Nord, 93430 Villetaneuse, France.

\begin{abstract}
This paper investigates the existence of minimizers for the so-called Kohn-Strang functional with affine boundary conditions. Such a functional, which arises in optimal shape design problems in electrostatics, is not quasi-convex, and therefore existence of minimizers is, in general, guaranteed only for its quasi-convex envelope. Such a quasi-convexification has been computed in two space dimensions in [11]. Recently, necessary and sufficient conditions on the affine boundary conditions for existence of minimizers for the Kohn-Strang functional have been derived in two space dimensions in [7]. We generalize these previous results for arbitrary space dimensions. Our method relies on the homogenization approach for relaxing optimal design problems. We also generalize our results to some variants of the Kohn-Strang functional.

(C) 1998 L'Association Publications de l'Institut Henri Poincaré. Published by Elsevier B.V. All rights reserved
\end{abstract}

Key words: homogenization, quasiconvexity, rank-one convexity, calculus of variations, relaxation, optimal design.

RÉSUMÉ. - Dans cet article nous étudions l'existence de minima pour la fonctionnelle dite de Kohn-Strang avec des conditions aux limites affines. Une telle fonctionnelle, issue de problèmes d'optimisation de formes en électrostatique, n'est pas quasiconvexe, et de ce fait l'existence de minima n'est en général garantie que pour son enveloppe quasiconvexe. Sa

Annales de l'Institut Henri Poincaré - Analyse non linéaire - 0294-1449 
quasiconvexification a été calculée en dimension deux d'espace par Kohn et Strang. Récemment, une condition nécessaire et suffisante sur la condition aux limites affine pour l'existence d'un minimum de la fonctionnelle de Kohn et Strang a été trouvée par Dacorogna et Marcellini. Nous généralisons ces résultats en toute dimension d'espace. Notre méthode repose sur la méthode d'homogénéisation pour relaxer des problèmes d'optimisation de formes. Nous donnons aussi quelques généralisations à des variantes de la fonctionnelle de Kohn et Strang.

(C) 1998 L'Association Publications de l'Institut Henri Poincaré. Published by Elsevier B.V. All rights reserved

\section{INTRODUCTION}

Let $\Omega$ be a bounded domain of $\mathbb{R}^{n}$. Let $u(x)$ be a vector-valued function from $\Omega$ into $\mathbb{R}^{N}$ with derivatives denoted by $D u=\left(\partial u_{i} / \partial x_{j}\right) \in \mathbb{R}^{n N}$. Let $\xi$ be a constant matrix in $\mathbb{R}^{n N}$, i.e., $\xi$ has $N$ lines and $n$ columns. Let $D_{\xi}$ denote the space

$$
D_{\xi}=\left\{\xi \cdot x+H_{0}^{1}\left(\Omega ; \mathbb{R}^{N}\right)\right\} .
$$

This paper is devoted to the question of existence of minimizers in $D_{\xi}$ for the following functional

$$
F(u)=\int_{\Omega} f(D u) d x,
$$

where the integrand $f$ is a function from $\mathbb{R}^{n N}$ into $\mathbb{R}^{+}$, defined by

$$
f(\eta)=\left\{\begin{array}{lr}
\lambda+\alpha|\eta|^{2}, & \eta \neq 0, \\
0, & \eta=0,
\end{array}\right.
$$

whith $0<\alpha, \lambda<+\infty$. In the case $n=2$, the function $f$ under consideration was introduced by Kohn and Strang in [11] as a model problem in the field of optimal design. Specifically, the associated minimization problem is equivalent to a shape optimization problem in electrostatics.

It is by now well-known that the functional $F$ is not (sequentially) weakly lower semi-continuous on $D_{\xi}$. Therefore, the direct method of the calculus of variations does not yield the existence of minimizers for (1) in $D_{\xi}$. Rather, one needs to introduce the relaxed functional (see [6])

$$
\bar{F}(u)=\int_{\Omega} Q f(D u) d x,
$$


where $Q f$ is the quasiconvex envelope of $f$ defined by

$$
Q f(\eta)=\inf _{\varphi \in H_{0}^{1}\left(Y ; \mathbb{R}^{N}\right)} \int_{Y} f(\eta+D \varphi) d y,
$$

where $Y=(0,1)^{n}$ is the unit cube of $\mathbb{R}^{n}$. Then, $u_{0}(x)=\xi \cdot x$ is a minimizer of the relaxed functional $\bar{F}$ on $D_{\xi}$, and

$$
Q f(\xi)=\inf _{u \in D_{\xi}} \frac{1}{|\Omega|} \int_{\Omega} f(D u) d x .
$$

When $n=2$, the quasiconvexification $Q f$ has been computed in [11]. The result is

$$
Q f(\eta)=\left\{\begin{array}{l}
\lambda+\alpha|\eta|^{2} \quad \text { if }|\eta|^{2}+2\left|a d j_{2} \eta\right| \geq \frac{\lambda}{\alpha}, \\
2 \sqrt{\alpha \lambda}\left(|\eta|^{2}+2\left|a d j_{2} \eta\right|\right)^{1 / 2}-2 \alpha\left|a d j_{2} \eta\right| \quad \text { otherwise }
\end{array}\right.
$$

where $a d j_{2} \eta$ is the $\frac{N(N-1)}{2}$ vector of the $2 \times 2$ minors of $\eta \in \mathbb{R}^{2 N}$.

In a recent paper [7] Dacorogna and Marcellini addressed the question of finding conditions for existence or non-existence of minimizers in $D_{\xi}$ of functionals of the type (1) for a general non-quasiconvex integrand $f$. As an example, they considered the Kohn-Strang energy, defined in (2), when $n=2$, and derived the following

THEOREM 1.1. - Let $\xi$ belong to $\mathbb{R}^{2 N}$. A necessary and sufficient condition for (1) to have a minimizer over $D_{\xi}$ is that, either $f(\xi)=Q f(\xi)$, or rank $\xi=2$.

The main results of the present paper are. generalizations of the computation of the quasi-convexification $Q f$ and of the above theorem to the case $n>2$. For arbitrary $n$, denoting by $\eta_{1}, \ldots, \eta_{n}$ the square roots of the eigenvalues of $\eta^{t} \eta$, we prove that (see Theorem 2.2)

$$
Q f(\eta)= \begin{cases}\alpha|\eta|^{2}+\lambda & \text { if } \sum_{i=1}^{n} \eta_{i} \geq \sqrt{\frac{\lambda}{\alpha}} \\ \alpha|\eta|^{2}-\alpha\left(\sum_{i=1}^{n} \eta_{i}\right)^{2}+2 \sqrt{\lambda \alpha} \sum_{i=1}^{n} \eta_{i} & \text { if } \sum_{i=1}^{n} \eta_{i}<\sqrt{\frac{\lambda}{\alpha}}\end{cases}
$$

Furthermore, for arbitrary $n$, we also prove (see Theorem 2.3)

THeOREM 1.2. - Let $\xi$ belong to $\mathbb{R}^{n N}$. A sufficient condition for (1) to have a minimizer over $D_{\xi}$ is that, either $f(\xi)=Q f(\xi)$, or rank $\xi=n$. While a sufficient condition for (1) to have no minimizer over $D_{\xi}$ is that $f(\xi)>Q f(\xi)$ and rank $\xi=1$.

Remark that our theorem does not furnish a necessary and sufficient condition for existence of minimizers, since it does not cover the case Vol. 15, $\mathrm{n}^{\circ} 3-1998$. 
$f(\xi)>Q f(\xi)$ and $2 \leq \operatorname{rank} \xi \leq n-1$. However, in such a case we conjecture there are no minimizers for (1) over $D_{\xi}$. To support our claim, we prove that in such a case there are no smooth-type minimizers of (1) in $D_{\xi}$ (see Proposition 2.5).

The existence of possible minimizers for (1) is not merely a question of purely theoretical interest. It also has important consequences in the context of optimal shape design. Let us briefly explore the connection between the Kohn-Strang energy, defined in (2), and optimal shape design (see Section 4 in [11] for more details). For each measurable subset $\omega$ of $\Omega$, define

$$
E(\omega, \xi)=\inf _{v \in D_{\xi, \omega}}\left\{\int_{\omega}\left(\alpha|D v(x)|^{2}+\lambda\right) d x\right\},
$$

where $D_{\xi, \omega}$ is the space defined by

$$
D_{\xi, \omega}=\left\{v \in D_{\xi} \text { s.t. } D v(x)=0 \text { a.e. in } \Omega \backslash \omega\right\} .
$$

Of course, any function $v \in D_{\xi, \omega}$ satisfies

$$
\int_{\Omega} f(D v) d x \leq \int_{\omega}\left(\alpha|D v(x)|^{2}+\lambda\right) d x
$$

but it is also true that

$$
E\left(\Omega_{v}, \xi\right) \leq \int_{\Omega} f(D v) d x
$$

where the measurable set $\Omega_{v} \subset \Omega$ is given by

$$
\Omega_{v}=\{x \in \Omega \text { s.t. } D v(x) \neq 0\} .
$$

Therefore, we deduce

$$
\inf _{\omega \subset \Omega} E(\omega, \xi)=\inf _{u \in D_{\xi}} \int_{\Omega} f(D u) d x .
$$

Whenever the right hand side of (4) admits a minimizer $u$, the corresponding set $\Omega_{u}$ minimizes the left hand side of (4). The minimization in the left hand side of (4) is a shape optimization problem in electrostatics : find the best arrangement of conductor $\alpha$ and holes so as to minimize the stored electrical energy. Since the seminal counter-examples of Murat [14] and Tartar [16], the generic non-existence of such optimal shapes is well-known. Rather, the problem is relaxed through the introduction, as admissible designs, of composite materials that mimic the behavior of minimizing sequences of 
shapes. Nevertheless, there could exist boundary conditions, corresponding to a special choice of $\xi$, for which an optimal shape is feasible. Our results provide conditions on $\xi$ that permit to assert or to rule out the existence of such an optimal shape.

As a final remark, we emphasize that our method is very specific to the type of functionals considered here, i.e., to the so-called Kohn-Strang energy and its various generalizations. Indeed, our key argument is the link between these functionals and the homogenization theory for two-phase composite materials. Of course, there are many other non-quasiconvex functionals for which existence of minimizers has been investigated : we refer to the recent article of Dacorogna and Marcellini [7] and references therein.

The outline of this paper is as follows. Section 2 is dedicated to the computation of $Q f(\eta)$ and to the proof of Theorem 1.2. Section 3 investigates the "dual" problem to (1)-(2), i.e., a functional acting on divergence-free fields. Our motivation in the analysis of this dual problem is twofold : firstly, the conditions for existence of minimizers are quite different, and secondly, it is this type of dual problem, and not (1)-(2) which arises in the context of optimal shape design (see [11] or [1] for details). Finally, section 4 deals with a partial generalization of Theorem 1.2 to the case of non-quadratic Kohn-Strang type functionals.

\section{EXISTENCE OF MINIMIZERS FOR THE KOHN-STRANG FUNCTIONAL}

This section is devoted to an analysis of possible minimizers for the functional

$$
\int_{\Omega} f(D u) d x
$$

where $\Omega$ is a bounded domain of $\mathbb{R}^{n}$, and $u$ is affine on the boundary of $\Omega$, i.e.,

$$
u \in D_{\xi}=\left\{\xi \cdot x+H_{0}^{1}\left(\Omega ; \mathbb{R}^{N}\right)\right\}, \quad \xi \in \mathbb{R}^{n N} .
$$

The specific function $f$ under consideration was introduced by Kohn and Strang in [11] as a model problem in the field of optimal design ; specifically, for $\eta \in \mathbb{R}^{n N}$,

$$
f(\eta)= \begin{cases}\lambda+\alpha|\eta|^{2}, & \eta \neq 0 \\ 0, & \eta=0\end{cases}
$$

where $0<\alpha, \lambda<+\infty$.

Vol. $15, \mathbf{n}^{\circ} 3-1998$. 
Only the case $n=2$ is investigated in [11]. In the two-dimensional setting it is shown in Section 4 of [11] that the minimization problem stems from a shape optimization problem in electrostatics.

In any case the functional defined in (5) is not (sequentially) weakly lower semi-continuous over $H^{1}\left(\Omega ; \mathbb{R}^{N}\right)$ so that minimizers for $(5)$ over $D_{\xi}$ defined in (6) need not exist. It was shown in [11], Theorem 1.1, that, when $n=2$, the quasiconvexification of $f$ is

$$
Q f(\eta)=\left\{\begin{array}{lr}
\lambda+\alpha|\eta|^{2} \text { if } & |\eta|^{2}+2\left|a d j_{2} \eta\right| \geq \frac{\lambda}{\alpha}, \\
2 \sqrt{\alpha \lambda}\left(|\eta|^{2}+2\left|a d j_{2} \eta\right|\right)^{1 / 2}-2 \alpha\left|a d j_{2} \eta\right| & \text { otherwise }
\end{array}\right.
$$

where $a d j_{2} \eta$ is the $\frac{N(N-1)}{2}$ vector of the $2 \times 2$ minors of $\eta \in \mathbb{R}^{2 N}$.

Accordingly the functional

$$
\int_{\Omega} Q f(D u) d x
$$

admits (a) minimizer(s) over $D_{\xi}$ and the minimum value of (9) coincides with the infimum of (5) over $D_{\xi}$ (see [11], Theorem 1.1).

As mentioned in the introduction, the following result about the existence of a minimizer for (5) (and not only for (9)) over $D_{\xi}$ is derived in [7], Theorem 6.1 :

THEOREM 2.1. - $(n=2)$ : A necessary and sufficient condition for (5) to have a minimizer over $D_{\xi}$ is that at least one of the following hold

(i) $\xi=0$,

(ii) $|\xi|^{2}+2\left|a d j_{2} \xi\right| \geq \frac{\lambda}{\alpha}$,

(iii) rank $\xi=2$.

The proof of Theorem 2.1 hinges on the knowledge of the quasiconvexification $Q f$ of $f$.

We prove below a generalization of Theorem 2.1 to arbitrary $n$. Our method is closely related to the homogenization approach for the relaxation of functional (5) because it uses decisively the characterization of $Q f(\eta)$ in terms of a finite dimensional minimization problem over the set of effective tensors associated to arbitrary mixtures of a material -with isotropic conductivity $\alpha$ - with voids of arbitrary shapes and sizes.

Let us begin with an explicit formula for the quasi-convexification $Q f(\eta)$ of the original function $f(\eta)$ for arbitrary $n$. 
THEOREM 2.2. - Let $0 \leq \eta_{1} \leq \ldots \leq \eta_{n}$ be the singular values of $\eta$ (i.e., the square roots of the eigenvalues of $\left.\eta^{t} \eta\right)$. Then

$$
Q f(\eta)= \begin{cases}\alpha|\eta|^{2}+\lambda & \text { if } \sum_{i=1}^{n} \eta_{i} \geq \sqrt{\frac{\lambda}{\alpha}} \\ \alpha|\eta|^{2}-\alpha\left(\sum_{i=1}^{n} \eta_{i}\right)^{2}+2 \sqrt{\lambda \alpha} \sum_{i=1}^{n} \eta_{i} & \text { if } \sum_{i=1}^{n} \eta_{i}<\sqrt{\frac{\lambda}{\alpha}}\end{cases}
$$

Furthermore, the quasi-convexification $Q f(\eta)$ coincides with the rank-one convex envelope of the original function $f(\eta)$.

Of course, in space dimension $n=2$ the definitions (8) and (10) of $Q f(\eta)$ are equivalent.

THEOREM 2.3. - Let $0 \leq \xi_{1} \leq \ldots \leq \xi_{n}$ be the singular values of $\xi$. A sufficient condition for (5), (7) to have a minimizer over $D_{\xi}$ is that at least one of the following hold

(i) $\xi=0$,

(ii) $\sum_{i=1}^{n} \xi_{i} \geq \sqrt{\frac{\lambda}{\alpha}}$,

(iii) $\operatorname{rank} \xi=n$,

while (5) has no minimizers over $D_{\xi}$ when

(iv) $\operatorname{rank} \xi=1$ and $|\xi|^{2}<\frac{\lambda}{\alpha}$.

Remark 2.4. - Note that sufficient conditions (i), (iv), and (ii) when rank $\xi=1$, were previously derived in Corollary 5.3 of [7]. Thus the new results are sufficient conditions (ii), when $2 \leq \operatorname{rank} \xi \leq n$, and (iii) for the existence of a minimizer. We however present a complete proof of Theorem 2.3 because our proof of (iv) as a sufficient condition for non existence does not use in an essential manner the rotational invariance of the original functional, in contrast with that given in [7].

Theorem 2.3 says nothing about the matrices $\xi$, with intermediate ranks between 2 and $n-1$, when $\sum_{i=1}^{n} \xi_{i}<\sqrt{\frac{\lambda}{\alpha}}$ (in contrast to the setting of Section 3 below). In such a case we conjecture that, for a bounded domain $\Omega$, there are no minimizers of (5), (7). To support our claim, we now state a partial result which rules out the existence of "smooth-type" minimizers in such a case.

Proposition 2.5. - Let $\Omega$ be a bounded domain with $|\partial \Omega|=0$. Let $0 \leq \xi_{1} \leq \ldots \leq \xi_{n}$ be the singular values of $\xi$. Assume that

$$
2 \leq \operatorname{rank} \xi \leq n-1, \text { and } \sum_{i=1}^{n} \xi_{i}<\sqrt{\frac{\lambda}{\alpha}}
$$


For each function $u \in D_{\xi}$, extended by $\xi \cdot x$ outside $\Omega$, define the set

$$
Z_{u}=\left\{x \in \mathbb{R}^{n} \text { such that } D u(x)=0\right\} .
$$

Then, (5) admits no minimizer $u \in D_{\xi}$ such that $Z_{u}$ is a closed set in $\Omega$.

Remark 2.6. - We must however confess our dissatisfaction with the condition on the closed character of $Z_{u}$. Indeed, it is doubtful whether this condition will be satisfied in general by a minimizer in $D_{\xi}$ (see, for example, the "confocal ellipsoïds" construction, when rank $\xi=n$, in the proof of Theorem 2.3). Nevertheless, we believe the idea of the proof of Proposition 2.5 interesting enough to be included here. We also refer to Remark 2.12 below for a discussion of this conjecture from a different perspective.

Proof of Theorem. 2.2. - The proof is divided into three steps. The first step provides a convenient characterization of the quasiconvexification of $f$ using homogenization theory. In the second step an explicit expression for $Q f$ is obtained. Finally the third step addresses the computation of the rank-one convex envelope of $f$ with the help of the Kohn-Strang algorithm (see section $5 \mathrm{C}$ in [11]).

STEP 1. - The starting idea in our proof is familiar in the context of homogenization whenever the microstructure exhibits voids. A poor conductor is allowed to fill those, which cures the degeneracy of the conductivity tensor and permits direct application of the theory of homogenization. Of course it still remains to show that the algebraic limit, as the conductivity of the poor conductor tends to 0 , of the obtained result is indeed the sought result (cf. for example [11], Section 6, or [1], Section 3 , for similar considerations).

We introduce, in lieu of (7),

$$
f_{\beta}(\eta)=\inf \left(\lambda+\alpha|\eta|^{2}, \beta|\eta|^{2}\right)
$$

where $1 \leq \beta<+\infty$. The sequential lower semicontinuous envclope of

$$
\int_{\Omega} f_{\beta}(D u) d x
$$

over $D_{\xi}$ is obtained by consideration of the new functional

$$
\int_{\Omega} Q f_{\beta}(D u) d x
$$


where $Q f_{\beta}$ is the quasiconvexification of $f_{\beta}$, i.e.,

$$
Q f_{\beta}(\eta)=\inf _{\varphi \in H_{\#}^{1}\left(Y ; \mathbb{F}^{N}\right)} \int_{Y} f_{\beta}(\eta+D \varphi) d y
$$

In (12) $Y$ is a unit cube in $\mathbb{R}^{n}\left(Y=(0,1)^{n}\right)$ and $H_{\#}^{1}\left(Y ; \mathbb{R}^{N}\right)$ denotes the subspace of $H^{1}\left(Y ; \mathbb{R}^{N}\right)$ of periodic functions. Note that the usual definition of the quasiconvexification of a functional over $\mathbb{R}^{n N}$ involves Dirichlet rather than periodic boundary data for the trial fields (see e.g. [6], Theorem 1.1, p. 201) but that both definitions are equivalent, at least when the functional is non negative, continuous and grows at most quadratically, which is precisely the case here ([5], Conjecture 3.7 and Theorem 3.1).

A simple switch in the minimizations leads to

$$
Q f_{\beta}(\eta)=\inf _{\chi \in L^{\infty}(Y ;\{0,1\})}\left\{A_{\chi} \eta^{t} \cdot \eta+\lambda \int_{Y} \chi d y\right\},
$$

where, denoting by $\left(e_{i}\right)_{1 \leq i \leq n}$ the canonical basis of $\mathbb{R}^{n}, A_{\chi}$ is a $n \times n$ symmetric matrix defined by its entries

$$
A_{\chi} e_{i} \cdot e_{j}=\inf _{\varphi \in H_{\#}^{1}(Y ; \mathbf{R})} \int_{Y}(\chi \alpha+(1-\chi) \beta)\left(e_{i}+D \varphi\right) \cdot\left(e_{j}+D \varphi\right) d y .
$$

The matrix $A_{\chi}$ is the limit in the sense of homogenization - the $H$-limit- of the sequence

$$
A_{\chi}^{n}=(\chi(n x) \alpha+(1-\chi(n x)) \beta) I_{2},
$$

where $I_{2}$ is the identity matrix on $\mathbb{R}^{n}$. See [15], Section 5 .

For a given $\theta \in L^{\infty}(\Omega ;[0,1])$, the set $\mathcal{G}_{\theta}^{\beta}$ of all possible $H$-limits of sequences of the form

$$
\left(\chi^{n}(x) \alpha+\left(1-\chi^{n}(x)\right) \beta\right) I_{2}
$$

with

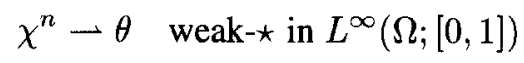

is known ([17], Theorem 1). It is of the form

$$
\mathcal{G}_{\theta}^{\beta}=\left\{A \in L^{\infty}\left(\Omega ; \mathbb{R}_{s}^{n^{2}}\right) \mid A(x) \in G_{\theta(x)}^{\beta} \text { a.e. }\right\}
$$

where $G_{\theta}^{\beta}$ is, for any $0 \leq \theta \leq 1$, a fixed set of matrices which is nothing else than the closure of the set of all $H$-limits of periodic sequences of Vol. 15, n n $^{\circ}-1998$. 
the form (14) with $\int_{Y} \chi d y=\theta$ (see [17], Proposition 3 for a constructive proof or [8] for a more general argument). Further, as proved in Theorem 1 of [17], $G_{\theta}^{\beta}$ is the set of all symmetric $n \times n$ matrices with eigenvalues $\lambda_{1}, \ldots, \lambda_{n}$ satisfying

$$
\left\{\begin{array}{l}
\underline{a}^{\beta}(\theta):=\left(\frac{\theta}{\alpha}+\frac{(1-\theta)}{\beta}\right)^{-1} \leq \lambda_{j} \leq \bar{a}^{\beta}(\theta):=\theta \alpha+(1-\theta) \beta, \quad 1 \leq j \leq n, \\
\sum_{j=1}^{n}\left(\lambda_{j}-\alpha\right)^{-1} \leq\left(\underline{a}^{\beta}(\theta)-\alpha\right)^{-1}+(n-1)\left(\bar{a}^{\beta}(\theta)-\alpha\right)^{-1}, \\
\sum_{j=1}^{n}\left(\beta-\lambda_{j}\right)^{-1} \leq\left(\beta-\underline{a}^{\beta}(\theta)\right)^{-1}+(n-1)\left(\beta-\bar{a}^{\beta}(\theta)\right)^{-1} .
\end{array}\right.
$$

Then (13) reads as

$$
Q f_{\beta}(\eta)=\inf _{0 \leq \theta \leq 1}\left\{f_{\beta}^{\star}(\theta, \eta)+\lambda \theta\right\}
$$

with

$$
f_{\beta}^{\star}(\theta, \eta)=\inf _{A \in G_{\theta}^{\beta}}\left\{A \eta^{t} \cdot \eta\right\} .
$$

Elementary order preserving properties of $H$ convergence immediately imply that $f_{\beta}^{\star}(\theta, \eta)$ is monotonically increasing with $\beta$. Furthermore, the function $f_{\beta}^{\star}$ can be checked to be continuous in both its arguments (cf. e.g. [9], Lemma 3.9 for a proof in a more general setting). Thus $Q f_{\beta}$ is a continuous function. Let us pause a moment in the proof of Theorem 2.2 in order to link $Q f_{\beta}$ to the quasiconvexification of $f$; this is the object of the next lemma.

LEMMA 2.7. - The sequence $Q f_{\beta}$ monotonically increases to $Q f$ as $\beta$ goes to $+\infty$, where $Q f$ is the quasiconvexification of $f$ defined in (7).

Proof. - Since $f_{\beta}$ monotonicity increases to $f$ as $\beta \nearrow^{+\infty}$,

$$
Q f_{\beta} \leq Q f .
$$

The functional $Q f$ is quasiconvex and has at most quadratic growth. It is thus rank-1 convex (cf. [6], p. 105), hence continuous (cf. [6], Theorem 2.3 , p. 29). Set

$$
g_{\beta}(\eta)=\min \left\{f_{\beta}(\eta), Q f(\eta)\right\}, \quad \eta \in \mathbb{R}^{n N} .
$$

The sequence $g_{\beta}$ is monotone in $\beta$ and continuous in $\eta$; it converges to $Q f$ as $\beta$ tends to $+\infty$. Dini's theorem implies that

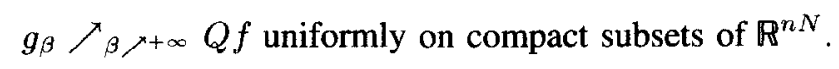


Note that

$$
Q g_{\beta} \leq Q f_{\beta} \leq Q f
$$

We prove that

$$
Q f \leq \lim _{\beta \rightarrow+\infty} Q g_{\beta}
$$

which establishes the desired result. Indeed take $\varphi$ in $H_{0}^{1}\left(Y ; \mathbb{R}^{N}\right)$ such that

$$
\begin{aligned}
Q g_{\beta}(\eta) & \geq \int_{Y} g_{\beta}(\eta+D \varphi) d y-\frac{1}{\beta} \\
& =\int_{Y} Q f(\eta+D \varphi) d y-\frac{1}{\beta}-\int_{Y}\left|g_{\beta}-Q f\right|(\eta+D \varphi) d y .
\end{aligned}
$$

Since $g_{\beta}(\eta)=Q f(\eta)$ as soon as $|\eta|$ is large enough (say $|\eta| \geq M$, $M$ independent of $\beta$ ), (18) implies that, denoting by $Y_{M}$ the set $\{y \in Y|| D \varphi(y)|\leq M+| \eta \mid\}$, for any $\varepsilon>0$,

$$
\int_{Y}\left|g_{\beta}-Q f\right|(\eta+D \varphi) d y=\int_{Y_{M}}\left|g_{\beta}-Q f\right|(\eta+D \varphi) d y \leq \varepsilon
$$

as soon as $\beta$ is large enough.

Thus, for $\beta$ large enough

$$
Q g_{\beta}(\eta) \geq \int_{Y} Q f(\eta+D \varphi) d y-\frac{1}{\beta}-\varepsilon \geq Q f(\eta)-\frac{1}{\beta}-\varepsilon
$$

because $Q f$ is quasiconvex. Letting $\varepsilon$ tend to 0 and $\beta$ to $+\infty$ proves (20), which concludes the proof of Lemma 2.7.

Let us resume the proof of Theorem 2.2. Upon setting

$$
f^{\star}(\theta, \eta)=\lim _{\beta \rightarrow+\infty} f_{\beta}^{\star}(\theta, \eta)
$$

we conclude, by virtue of Lemma 2.7 , that

$$
Q f(\eta)=\inf _{0 \leq \theta \leq 1}\left\{f^{\star}(\theta, \eta)+\lambda \theta\right\} .
$$

Since $f_{\beta}^{\star}$ is continuous in $\theta$, there exists, for a fixed $\beta$, a value $\theta_{\beta}$ of $\theta$ such that

$$
Q f_{\beta}(\eta)=f^{\star}\left(\theta_{\beta}, \eta\right)+\lambda \theta_{\beta} .
$$


Extract a converging subsequence of $\theta_{\beta}\left(0 \leq \theta_{\beta} \leq 1\right)$, still indexed by $\beta$, and call $\bar{\theta}$ the limit. If $\beta<\beta^{\prime}$, the monotone character of $f_{\beta}^{\star}$ implies

$$
Q f(\eta) \geq Q f_{\beta^{\prime}}(\eta)=f_{\beta^{\prime}}^{\star}\left(\theta_{\beta^{\prime}}, \eta\right)+\lambda \theta_{\beta^{\prime}} \geq f_{\beta}^{\star}\left(\theta_{\beta^{\prime}}, \eta\right)+\lambda \theta_{\beta^{\prime}} .
$$

We let $\beta^{\prime}$ tend to $+\infty$ and obtain, by virtue of the continuous character of $f_{\beta}^{\star}(\cdot, \eta)$,

$$
Q f(\eta) \geq f_{\beta}^{\star}(\bar{\theta}, \eta)+\lambda \bar{\theta}
$$

Thus, letting $\beta$ tend to $+\infty$,

$$
Q f(\eta) \geq f^{\star}(\bar{\theta}, \eta)+\lambda \bar{\theta}
$$

i.e.,

$$
Q f(\eta)=f^{\star}(\bar{\theta}, \eta)+\lambda \bar{\theta}
$$

and we conclude from (21), (22) that

$$
Q f(\eta)=\min _{0 \leq \theta \leq 1}\left\{f^{\star}(\theta, \eta)+\lambda \theta\right\}=\lim _{\beta \nearrow^{+\infty}}\left(\min _{0 \leq \theta \leq 1}\left\{f_{\beta}^{\star}(\theta, \eta)+\lambda \theta\right\}\right) .
$$

A much more explicit expression for $f^{\star}(\theta, \eta)$ may be derived with the help of (17) which defines $\int_{\beta}^{\star}(\theta, \eta)$ as the infimum of a linear functional over the set $G_{\theta}^{\beta}$. In view of equation (15), let us define $G_{\theta}^{\infty}$ as the algebraic limit, as $\beta \nearrow^{+\infty}$, of $G_{\theta}^{\beta}$, i.e., the set of symmetric $n \times n$ matrices with (possibly infinite) eigenvalues $\lambda_{1}, \ldots, \lambda_{n}$ satisfying

$$
\left\{\begin{array}{l}
\lambda_{i} \geq \frac{\alpha}{\theta}, \quad 1 \leq i \leq n \\
\sum_{i=1}^{n}\left(\lambda_{i}-\alpha\right)^{-1} \leq \frac{\theta}{(1-\theta) \alpha}
\end{array}\right.
$$

This yields

$$
f^{\star}(\theta, \eta)=\inf _{A \in G_{\theta}^{\infty}}\left\{A \eta^{t} \cdot \eta\right\},
$$

which completes the first step in the proof.

STEP 2. - To compute $f^{\star}(\theta, \eta)$, we first remark that

$$
f^{\star}(\theta, \eta)=\inf _{A \in G_{\theta}^{\infty}} \operatorname{tr}(A H)
$$

where $H=\eta^{t} \eta$ is a $n \times n$ symmetric matrix. Let us denote by $0 \leq \eta_{1} \leq \ldots \leq \eta_{n}$ the singular values of $\eta$, i.e., the square roots of 
the eigenvalues of $H$. A well-known result of von Neumann (see e.g. [13]) yields

$$
f^{\star}(\theta, \eta)=\inf _{\lambda_{1} \leq \ldots \leq \lambda_{n}}\left\{\sum_{i=1}^{n} \lambda_{i} \eta_{n+1-i}^{2}\right\},
$$

where $\left(\lambda_{1}, \ldots, \lambda_{n}\right)$ are the ordered eigenvalues of $A$, and the infimum is taken on the convex set defined in (24). Therefore, the infimum in (25) is a minimum if infinite values of $\lambda_{i}$ are allowed. If the minimizer in (25) is such that, for some index $i_{0}$,

$$
\lambda_{i_{0}}=\frac{\alpha}{\theta},
$$

then, the constraints in (25) implies that all the others eigenvalues $\lambda_{i}$ are infinite for $i \neq i_{0}$. This can happen only if $\eta_{i}=0$ for $i \neq n+1-i_{0}$, i.e., if $\eta$ has rank one. Let us assume for the moment that the rank of the tensor $\eta$ is strictly greater than one. Then, when writing the optimality conditions for minimizers in (25), the constraints $\lambda_{i} \geq \frac{\alpha}{\theta}$, which are not saturated, do not play any part. Therefore, the optimality conditions read

$$
\exists C>0 \text { such that } \eta_{n+1-i}^{2}=\frac{C^{2}}{\left(\lambda_{i}-\alpha\right)^{2}} 1 \leq i \leq n .
$$

Taking into account that, at the minimum, $\sum_{i=1}^{n}\left(\lambda_{i}-\alpha\right)^{-1}=\frac{\theta}{(1-\theta) \alpha}$, a straightforward calculation yields

$$
C=\frac{(1-\theta) \alpha}{\theta} \sum_{i=1}^{n} \eta_{i},
$$

and

$$
f^{\star}(\theta, \eta)=\alpha \sum_{i=1}^{n} \eta_{i}^{2}+\frac{(1-\theta) \alpha}{\theta}\left(\sum_{i=1}^{n} \eta_{i}\right)^{2} .
$$

Formula (27) is immediately seen to hold true also if $\eta$ has rank one. Then, a simple minimization over $\theta$ in (23) leads to the desired formula (10).

STEP 3. - According to Kohn and Strang [11], section 5C, the rank-one convex envelope $R f$ of $f$, i.e., the largest rank-one convex function below $f$, is the limit as $p$ goes to infinity of the sequence of functions $f^{p}$ defined by

$$
f^{0}(\eta)=f(\eta), \quad f^{p+1}(\eta)=\inf _{\substack{\eta=\theta \eta_{1}+(1-\theta) \eta_{2}, 0 \leq \theta \leq 1 \\ \operatorname{rank}_{\left(\eta_{1}-\eta_{2}\right) \leq 1}}}\left(\theta f^{p}\left(\eta_{1}\right)+(1-\theta) f^{p}\left(\eta_{2}\right)\right) .
$$


The precise computation of $\lim _{p \rightarrow+\infty} f^{p}$ is cumbersome. Rather, we construct a sequence $f \geq g^{p} \geq f^{p}$ such that $g^{n}=Q f$. Since $Q f$ is a rank-one convex function and $f^{p}$ monotonically decreases to $R f$, this proves that $g^{n}=f^{n}=Q f=R f$. Let us define the sequence $\left(g^{p}\right)_{0 \leq p \leq n}$ by

$$
g^{p}(\eta)=\left\{\begin{array}{lr}
\alpha|\eta|^{2}-\alpha\left(\sum_{i=1}^{n} \eta_{i}\right)^{2}+2 \sqrt{\lambda \alpha} \sum_{i=1}^{n} \eta_{i} \\
\alpha|\eta|^{2}+\lambda & \text { if } \sum_{i=1}^{n} \eta_{i}<\sqrt{\frac{\lambda}{\alpha}} \text { and } \operatorname{rank}(\eta) \leq p \\
\text { otherwise }
\end{array}\right.
$$

Obviously $g^{p}$ is a decreasing sequence such that $g^{0}=f$ and $g^{n}=Q f$. It remains to prove that $g^{p} \geq f^{p}$ for $0 \geq p \geq n$. We proceed by induction on $p$. It is true for $p=0$; let us assume it is also true up to order $p$. Then $g^{p+1}(\eta)=g^{p}(\eta)$ whenever the rank of $\eta$ is different from $p+1$. Thus $g^{p+1}(\eta) \geq f^{p+1}(\eta)$ if $\operatorname{rank}(\eta) \neq p+1$. When $\operatorname{rank}(\eta)=p+1$, the polar decomposition of $\eta$ allows us to write

$$
\eta=\sum_{i=1}^{p+1} \eta_{i} e_{i} \otimes h_{i}
$$

where $\left(\eta_{i}\right)$ are the singular values of $\eta$ and $\left(e_{i}\right),\left(h_{i}\right)$ are orthonormal families of vectors in $\mathbb{R}^{N}$ and $\mathbb{R}^{n}$ respectively. Then,

$$
\begin{aligned}
f^{p+1}(\eta) & \leq \inf _{0 \leq \theta \leq 1}\left(\theta f^{p}\left(\eta_{1}\right)+(1-\theta) f^{p}\left(\eta_{2}\right)\right) \\
& \leq \inf _{0 \leq \theta \leq 1}\left(\theta g^{p}\left(\eta_{1}\right)+(1-\theta) g^{p}\left(\eta_{2}\right)\right),
\end{aligned}
$$

where

$\eta_{1}=\eta+\frac{1-\theta}{\theta} \eta_{p+1} e_{p+1} \otimes h_{p+1}, \quad$ and $\quad \eta_{2}=\eta-\eta_{p+1} e_{p+1} \otimes h_{p+1}$.

Since the rank of $\eta_{1}$ is $p+1, g^{p}\left(\eta_{1}\right)=f\left(\eta_{1}\right)$, and a tedious minimization over $\theta$ yields

$$
g^{p+1}(\eta)=\inf _{0 \leq \theta \leq 1}\left(\theta g^{p}\left(\eta_{1}\right)+(1-\theta) g^{p}\left(\eta_{2}\right)\right)
$$

which proves that $f^{p+1} \leq g^{p+1}$ for any $p$. This completes the proof of Theorem 2.2.

Remark 2.8. - In [11], section 5C, Kohn and Strang already proved, in the case $n=2$, that $Q f$ coincides with the rank-one convex envelope of $f$. Our proof that it is also true in higher dimensions $n \geq 2$ is a generalization 
of their two-dimensional proof, once the quasiconvexification $Q f$ of $f$ has been computed.

Remark 2.9. - Our computation of the quasiconvex envelope $Q f$ does not use in an essential manner the knowledge of the entire $G$-closure, i.e., the set $G_{\theta}^{\infty}$ (see (15)). It is enough to be able to minimize $\left\{A \eta^{t} \cdot \eta\right\}$ over all $A^{\prime}$ 's in $G_{\theta}^{\infty}$ (see the second step in the proof of Theorem 2.2). At the price of tedious computations, this latter task can be performed without the explicit knowledge of $G_{\theta}^{\infty}$ by using the so-called Hashin-Shtrikman variational principle (see [3] or [2]).

Proof of Theorem. 2.3. - If $\xi=0$ or $\sum_{i=1}^{n} \xi_{i} \geq \sqrt{\frac{\lambda}{\alpha}}$, then $f(\xi)=Q f(\xi)$ which proves that $\xi \cdot x$ is a minimizer for (5) over $D_{\xi}$. The rest of the proof is divided into two steps. The first step addresses the case where rank $\xi=1$ while the second one examines the case where rank $\xi=n$.

STEP 1. - If rank $\xi=1$, we deduce from Theorem 2.2 (or more precisely from (23)) that

$$
Q f(\xi)=\min _{0 \leq \theta \leq 1}\left\{\frac{\alpha}{\theta}|\xi|^{2}+\lambda \theta\right\}
$$

Either $\alpha|\xi|^{2} \geq \lambda$, in which case the minimum is obtained for $\theta=1$ and

$$
Q f(\xi)=\alpha|\xi|^{2}+\lambda=f(\xi),
$$

which proves sufficient condition ii) for the existence of a minimizer. Or $\alpha|\xi|^{2}<\lambda$ in which case the minimum is obtained for

$$
\theta_{\min }=\left(\frac{\alpha}{\lambda}\right)^{1 / 2}|\xi|,
$$

and

$$
Q f(\xi)=2(\alpha \lambda)^{1 / 2}|\xi| .
$$

In such a case assume that $u$ is a minimizer for (5) over $D_{\xi}$. Since, by the very definition of the quasiconvexification $Q f$ of $f, \xi . x$ is a minimizer for

$$
\int_{\Omega} Q f(D u) d x
$$

over $D_{\xi}$,

$$
Q f(\xi)=\frac{1}{|\Omega|} \int_{\Omega} f(D u) d x
$$


Define the set

$$
\Omega_{u}=\{x \in \Omega \mid D u(x) \neq 0\} .
$$

Then

$$
Q f(\xi)=\frac{\alpha}{|\Omega|} \int_{\Omega_{u}}|D u(x)|^{2} d x+\lambda \frac{\left|\Omega_{u}\right|}{|\Omega|} .
$$

Note that

$$
\frac{1}{|\Omega|} \int_{\Omega} D u d x=\xi=\frac{\left|\Omega_{u}\right|}{|\Omega|} \frac{1}{\left|\Omega_{u}\right|} \int_{\Omega_{u}} D u d x .
$$

Jensen's inequality implies, in view of (29), (30), that

$$
\begin{aligned}
Q f(\xi) & \geq \frac{\alpha\left|\Omega_{u}\right|}{|\Omega|}\left(\frac{1}{\left|\Omega_{u}\right|} \int_{\Omega_{u}} D u(x) d x\right)^{2}+\lambda \frac{\left|\Omega_{u}\right|}{|\Omega|} \\
& =\alpha \frac{|\Omega|}{\left|\Omega_{u}\right|}|\xi|^{2}+\lambda \frac{\left|\Omega_{u}\right|}{|\Omega|}
\end{aligned}
$$

which, together with (28), implies that

$$
\theta_{\min }=\frac{\left|\Omega_{u}\right|}{|\Omega|},
$$

and

$$
\frac{1}{|\Omega|} \int_{\Omega}|D u(x)|^{2} d x=\frac{|\Omega|}{\left|\Omega_{u}\right|}|\xi|^{2},
$$

or, equivalently,

$$
\int_{\Omega_{u}}|D u(x)|^{2} d x=\frac{|\Omega|^{2}}{\left|\Omega_{u}\right|}|\xi|^{2}
$$

If $\nu$ is a fixed element of $\mathbb{R}^{n N}$, (31) leads to

$$
\int_{\Omega_{u}}|D u(x)-\nu|^{2} d x=\frac{|\Omega|^{2}}{\left|\Omega_{u}\right|}\left|\xi-\nu \frac{\left|\Omega_{u}\right|}{|\Omega|}\right|^{2},
$$

and a choice of $\nu=\frac{|\Omega|}{\left|\Omega_{u}\right|} \xi$ yields

$$
\int_{\Omega_{u}}\left|D u(x)-\frac{|\Omega|}{\left|\Omega_{u}\right|} \xi\right|^{2} d x=0 .
$$


Consequently,

$$
D u(x)= \begin{cases}\frac{|\Omega|}{\left|\Omega_{u}\right|} \xi, & \text { a.e. on } \Omega_{u}, \\ 0, & \text { a.e. on } \Omega \backslash \Omega_{u} .\end{cases}
$$

But a vector valued function cannot have a gradient that only takes two values on $\Omega$ unless $\Omega_{u}$ and $\Omega \backslash \Omega_{u}$ are made of parallel strips normal to their difference (cf. Proposition 1 of [4]). These layers necessarily meet the boundary of $\Omega$ and the boundary value of $u$ cannot be affine all along that boundary. There are thus no minimizers $u$ for (5) over $D_{\xi}$. The proof of sufficient condition iv) for the non existence of minimizers is complete.

STEP 2. - If rank $\xi=n$, then $H=\xi^{t} \xi$ is a positive definite $n \times n$ matrix. Denote by $0<\xi_{1}^{2} \leq \ldots \leq \xi_{n}^{2}<+\infty$ the eigenvalues of $H$.

By virtue of (23)

$$
Q f(\xi)=f^{\star}(\theta, \xi)+\lambda \theta
$$

for some $\theta \in[0,1]$. Further $\theta \neq 0$ otherwise

$$
Q f(\xi)=f^{\star}(0, \xi)=\lim _{\beta \rightarrow+\infty} f_{\beta}^{\star}(0, \xi)=\lim _{\beta \rightarrow+\infty} \beta|\xi|^{2}=+\infty
$$

because $\xi \neq 0$.

If $\theta=1$ then

$$
f^{\star}(1, \xi)=\alpha|\xi|^{2}
$$

and

$$
Q f(\xi)=\alpha|\xi|^{2}+\lambda
$$

from which it is immediately concluded that $\xi \cdot x$ is a minimizer for (5) over $D_{\xi}$.

Assume from now onward that $\theta \notin\{0,1\}$ and recall from (25) that

$$
f^{\star}(\theta, \xi)=\inf _{A \in G_{\theta}^{\infty}} \operatorname{tr}(A H)=\inf _{\lambda_{1} \leq \ldots \leq \lambda_{n}}\left\{\sum_{j=0}^{n-1} \xi_{n-j}^{2} \lambda_{j+1}\right\},
$$

where the eigenvalues $\lambda_{j}$ belong to the convex set defined in (24).

The infimum in (32) is attained and this at a point $\left(\lambda_{1}^{0}, \ldots, \lambda_{n}^{0}\right)$ such that

$$
\left\{\begin{array}{l}
\lambda_{j}^{0}>\frac{\alpha}{\theta}, \quad 1 \leq j \leq n, \\
\sum_{j=1}^{n}\left(\lambda_{j}^{0}-\alpha\right)^{-1}=\frac{\theta}{(1-\theta) \alpha},
\end{array}\right.
$$

since rank $\xi$ is strictly greater than one (cf. step 2 in the proof of Theorem 2.2). 
We now revisit the explicit construction proposed in Proposition 6 of [17] with the help of the so-called coated ellipsoïd. Consider two ellipsoïds $B_{\rho^{-}}$and $B_{\rho^{+}}$with equations

$\sum_{j=1}^{n} \frac{x_{j}^{2}}{\rho^{ \pm}+m_{j}}=1, \rho^{-}<\rho^{+}, m=\left(m_{1}, \ldots, m_{n}\right) \in \mathbb{R}^{n}, \rho^{-}+\inf _{j}\left(m_{j}\right)>0$.

In (33) $x_{j}$ denotes the $j^{\text {th }}$ component of $x$ in the orthonormal basis $\left\{e_{i}\right\}_{i=1, \ldots, n}$ generated by the eigendirections of $H$.

For each vector $\zeta \in \mathbb{R}^{n}$, the real-valued solution $u\left(\zeta, B_{\rho^{+}}\right)$of

$$
\begin{cases}-\operatorname{div}\left(A(x) D u\left(\zeta, B_{\rho^{+}}\right)\right)=0 & \text { on } \mathbb{R}^{n} \backslash B_{\rho^{-}} \\ u\left(\zeta, B_{\rho^{+}}\right)=0 & \text { on } \partial B_{\rho^{-}} \\ u\left(\zeta, B_{\rho^{+}}\right)=\zeta \cdot x & \text { on } \mathbb{R}^{n} \backslash B_{\rho^{+}}\end{cases}
$$

with

$$
A(x)= \begin{cases}\alpha I & \text { on } B_{\rho^{+}} \backslash B_{\rho^{-}}, \\ \sum_{i=1}^{n} \lambda_{i} e_{i} \otimes e_{i} & \text { on } \mathbb{R}^{n} \backslash B_{p^{+}}, \frac{\alpha}{\theta}<\lambda_{1} \leq \ldots \leq \lambda_{n}<+\infty,\end{cases}
$$

is of the form (see Proposition 6 of [17])

$$
u\left(\zeta, B_{\rho^{+}}\right)(x)=\sum_{i=1}^{n} \zeta_{i} x_{i} f_{i}(\rho)
$$

with

$$
f_{i}(\rho)=\left[\int_{\rho^{-}}^{\rho^{+}} \frac{d t}{g_{m}(t)\left(t+m_{i}\right)}\right]^{-1} \int_{\rho^{-}}^{\rho} \frac{d t}{g_{m}(t)\left(t+m_{i}\right)}, 1 \leq i \leq n .
$$

In (36)

$$
g_{m}(\rho)=\prod_{k=1}^{n}\left(\rho+m_{k}\right)^{1 / 2}
$$

is the volume of the ellipsoïd $B_{\rho}$ with equation

$$
\sum_{j=1}^{n} \frac{x_{j}^{2}}{\rho+m_{j}}=1
$$

Further

$$
\sum_{i=1}^{n} \frac{1}{\lambda_{i}-\alpha}=\left(\frac{g_{m}\left(\rho_{+}\right)}{g_{m}\left(\rho^{-}\right)}-1\right) \frac{1}{\alpha}
$$


so that, if we choose $\rho_{-} / \rho_{+}$in a manner such that

$$
g_{m}\left(\rho_{-}\right)=(1-\theta) g_{m}\left(\rho_{+}\right)
$$

we get

$$
\sum_{i=1}^{n} \frac{1}{\lambda_{i}-\alpha}=\frac{\theta}{(1-\theta) \alpha}, \quad \frac{\alpha}{\theta}<\lambda_{1} \leq \ldots \leq \lambda_{n}<+\infty .
$$

Finally when $m$ spans $\mathbb{R}_{+}^{n}$, all points $\left(\lambda_{1}, \ldots, \lambda_{n}\right)$ satisfying (39) can be obtained as can be shown through a degree argument (see Proposition 6 of [17]). Before completing the proof of Theorem 2.3, we make a few useful comments on the above construction.

Remark 2.10. - The ellipsoïds $B_{\rho^{ \pm}}$corresponding to a given point $\left(\lambda_{1}, \ldots, \lambda_{n}\right)$ satisfying (38) can always be rescaled through multiplication of $\rho^{+}, \rho^{-}$and the $m_{i}$ 's by a small number so that $B_{\rho^{+}}$lies inside the unit cube $Q$. Then

$$
\begin{aligned}
\alpha \int_{B_{\rho^{+}}} & \left|D u\left(\zeta, B_{\rho^{+}}\right)\right|^{2} d x \\
= & \alpha \int_{\partial B_{\rho^{+}}} u \frac{\partial u}{\theta n} d H^{n-1} \\
& =-\int_{\partial\left(\mathbf{R}^{n}-B_{\rho^{+}}\right)} u A D u \cdot \vec{n} d H^{n-1} \\
& =\int_{\partial Q} u A D u \cdot \vec{n} d H^{n-1}-\int_{Q / B_{\rho^{+}}} A D u \cdot D u d x
\end{aligned}
$$

where, in the last two equalities, $\vec{n}$ represents the outward normal to the hypersurface over which integration is performed. But, according to the third equality in (34), $u=\zeta \cdot x$ on $\bar{Q} \backslash B_{\rho^{+}}$so that

$$
\begin{cases}\int_{\partial Q} u A D u \cdot \vec{n} d H^{n-1} & =\sum_{j=1}^{n} \lambda_{j} \zeta_{j}^{2}, \\ \int_{Q \backslash B_{\rho}} A D u \cdot D u d x & =\left(\sum_{j=1}^{n} \lambda_{j} \zeta_{j}^{2}\right)\left(1-\left|B_{\rho^{+}}\right|\right) .\end{cases}
$$

Thus

$$
\alpha \int_{B_{\rho^{+}}}\left|D u\left(\zeta, B_{\rho^{+}}\right)\right|^{2} d x=\left(\sum_{j=1}^{n} \lambda_{j} \zeta_{j}^{2}\right)\left|B_{\rho^{+}}\right| .
$$

Remark 2.11. - Everywhere inside $B_{\rho^{+}} \backslash B_{\rho^{-}}$one has

$$
D u\left(\zeta, B_{\rho^{+}}\right)(x) \neq 0 .
$$


Indeed, by virtue of the form (35) of $u\left(\zeta, B_{\rho^{+}}\right)$,

$$
\frac{\partial u}{\partial x_{j}}=\zeta_{j} f_{j}(\rho)+\sum_{i=1}^{n} \zeta_{i} x_{i} f_{i}^{\prime}(\rho) \frac{\partial \rho}{\partial x_{j}} .
$$

But, from (37),

$$
\sum_{i=1}^{n} \frac{x_{i}^{2}}{\left(\rho+m_{i}\right)^{2}} \frac{\partial \rho}{\partial x_{j}}=2 \frac{x_{j}}{\rho+m_{j}}
$$

thus

$$
\frac{\partial u}{\partial x_{j}}=\zeta_{j} f_{j}(\rho)+\frac{2}{\left(\sum_{i=1}^{n} \frac{x_{i}^{2}}{\left(\rho+m_{i}\right)^{2}}\right)} \sum_{i=1}^{n} \zeta_{i} x_{i} f_{i}^{\prime}(\rho) \frac{x_{j}}{\left(\rho+m_{j}\right)} .
$$

If

$$
\frac{\partial u}{\partial x_{j}}=0, \quad j=1, \ldots, n
$$

it implies that

$$
\begin{aligned}
\sum_{j=1}^{n} \zeta_{j}\left(\rho+m_{j}\right) f_{j}^{\prime}(\rho) \frac{\partial u}{\partial x_{j}}= & \sum_{j=1}^{n} \zeta_{j}^{2}\left(\rho+m_{j}\right) f_{j}^{\prime}(\rho) f_{j}(\rho) \\
& +\frac{2\left(\sum_{i=1}^{n} \zeta_{i} x_{i} f_{i}^{\prime}(\rho)\right)^{2}}{\left(\sum_{i=1}^{n} \frac{x_{i}^{2}}{\left(\rho+m_{i}\right)^{2}}\right)}=0
\end{aligned}
$$

which is impossible since $f_{j}(\rho)$ and $f_{j}^{\prime}(\rho)$ are positive for $\rho^{-}<\rho<\rho^{+}$.

Coming back to the proof of Theorem 2.3, we consider the point $\left(\lambda_{1}^{0}, \ldots, \lambda_{n}^{0}\right)$ satisfying (38) such that the infimum in (32) is attained. According to Remark 2.10, there exists, say for a given $\rho^{-}$(hence a given $\rho^{+}$determined by (38)), rescaled versions of $B_{\rho^{-}}$and $B_{\mu^{+}}$lying inside the unit cube and corresponding to $\left(\lambda_{1}^{0}, \ldots, \lambda_{n}^{0}\right)$. Vitali's covering theorem implies the existence of a countable family $\mathcal{G}$ of disjoint homothetics, of ratio less than or equal to 1 , of $B_{\rho^{+}}$such that

$$
\left|\Omega-\bigcup_{B^{+} \in \mathcal{G}} B^{+}\right|=0
$$

Define, for $x \in \Omega$,

$$
u(\zeta)(x)= \begin{cases}u\left(\zeta, B^{+}\right)(x) & \text { if } x \in B^{+}, B^{+} \in \mathcal{G} \\ \zeta \cdot x & \text { otherwise }\end{cases}
$$


Then, according to Remark 2.10 , for $B^{+}$in $\mathcal{G}$,

$$
\alpha \int_{B^{+}}|D u(\zeta)|^{2} d x=\left(\sum_{j=1}^{n} \lambda_{j} \zeta_{j}^{2}\right)\left|B^{+}\right| .
$$

Thus, by virtue of (40),

$$
\alpha \int_{\Omega}|D u(\zeta)|^{2} d x=\left(\sum_{j=1}^{n} \lambda_{j} \zeta_{j}^{2}\right)|\Omega| .
$$

Choose $\zeta$ to be successively $\xi_{1}, \ldots, \xi_{N}$ (the lines of the matrix $\xi$ ), and define

$$
u_{\xi}=\left(u\left(\xi_{1}\right), \ldots, u\left(\xi_{N}\right)\right) .
$$

Then, in view of Remark 2.11 , and upon denoting by $B^{-}$the homothetics of $B_{p^{-}}, u_{\xi}$, an element of $D_{\xi}$, satisfies

$$
\begin{aligned}
u_{\xi}(x) & =0 \text { on } B^{-} \quad\left(B^{-} \subset B^{+}, B^{+} \in \mathcal{G}\right), \\
D u_{\xi}(x) & \neq 0 \text { on } B^{+} \quad B^{+} \in \mathcal{G},
\end{aligned}
$$

hence

$$
\begin{aligned}
\int_{\Omega} f\left(D u_{\xi}(x)\right) d x & =\left(\sum_{i=1}^{N} \sum_{j=1}^{n} \lambda_{j} \xi_{j i}^{2}\right)|\Omega|+\lambda \sum_{B^{+} \in \mathcal{G}}\left|B^{+} \backslash B^{-}\right| \\
& =(\operatorname{tr}(A H)+\lambda \theta)|\Omega| \\
& =\left(\sum_{j=0}^{n-1} \xi_{n-j}^{2} \lambda_{j+1}^{0}+\lambda \theta\right)|\Omega|=Q f(\xi)|\Omega| .
\end{aligned}
$$

But

$$
\inf \left\{\int_{\Omega} f(D u(x)) d x \mid u \in D_{\xi}\right\}=Q f(\xi)|\Omega|,
$$

which in view of (43) permits us to conclude that $u_{\xi}$ is a minimizer for (5) over $D_{\xi}$ and proves (iii) in Theorem 2.3. The proof of that theorem is complete.

Remark 2.12. - Let us examine briefly the confocal ellipsoïds construction adapted to a matrix $\xi$ of rank $p$ with $2 \leq p \leq n-1$. Let us denote by $0=\xi_{1}^{2}=\ldots=\xi_{n-p}^{2}<\xi_{n-p+1}^{2} \leq \ldots \leq \xi_{n}^{2}<+\infty$ the eigenvalues of the matrix $H=\xi^{t} \xi$, and by $e_{1}, \ldots, e_{n}$ the corresponding eigendirections. In the computation of $f^{\star}(\theta, \xi)$, the optimality condition (26) in the minimization over the eigenvalues $\left(\lambda_{i}\right)_{1 \leq i \leq n}$ of the homogenized 
tensor implies that the $(n-p)$ largest eigenvalues $\lambda_{i}$ are equal to $+\infty$. According to Proposition 6 in [17] the corresponding values of the ellipsoïds parameters $m_{i}$ are also equal to $+\infty$, implying that the domain $B_{\rho^{-}}$and $B_{\rho^{+}}$defined by equation (33) are cylinders obtained by translation in the directions $e_{1}, \ldots, e_{n-p}$ of $p$-dimensional ellipsoïds with axes given by $e_{n-p+1}, \ldots, e_{n}$. It can easily be checked that a solution $u\left(\zeta, B_{p^{+}}\right)$of (34) can still be defined, which does not depend on the variables $x_{1}, \ldots, x_{n-p}$. Therefore, Step 2 of the proof of Theorem 2.3 can be generalized if we assume that $\Omega$ is a cylindric (unbounded) domain defined by translation in the $e_{1}, \ldots, e_{n-p}$ directions of a $p$-dimensional domain in the subspace generated by $e_{n-p+1}, \ldots, e_{n}$. In other words, this proves the existence of a minimizer for (5) when $2 \leq \operatorname{rank} \xi \leq n-1$ and $\sum_{i=1}^{n} \xi_{i}<\frac{\lambda}{\alpha}$ if the domain $\Omega$ is an unbounded cylindrical domain, aligned with some of the eigendirections of $H=\xi^{t} \xi$.

However, when $\Omega$ has no such special properties (in particular if it is a bounded domain), we conjecture that there exists no minimizer for (5) over $D_{\xi}$ when $2 \leq \operatorname{rank} \xi \leq n-1$ and $\sum_{i=1}^{n} \xi_{i}<\frac{\lambda}{\alpha}$. We make such a claim because we believe that the optimality condition (26), which forces the $(n-\operatorname{rank} \xi)$ largest eigenvalues $\lambda_{i}$ of the homogenized tensor to be equal to $+\infty$, implies that possible minimizers do not depend on the corresponding ( $n$-rank $\xi$ ) variables $x_{i}$, a fact that would violate the boundary condition.

Proof of Proposition 2.5. - Let $u \in D_{\xi}$ be a minimizer for (5) such that $Z_{u}$ is closed in $\Omega$. Let $d=d\left(Z_{u}, \partial \Omega\right)$ denote the Euclidean distance between $Z_{u}$ and $\partial \Omega$. Since $D u(x)=\xi \neq 0$ for all points $x$ of the closed set $\mathbb{R}^{n} \backslash \Omega$, the distance $d$ is strictly positive. This implies the existence, for any boundary condition on $\partial \Omega$, of a test function, satisfying the boundary condition, and such that its gradient vanishes on $Z_{u}$. In other words, for any $u_{0} \in H^{1}(\Omega)$,

$$
\inf _{\substack{\phi \in\left\{u_{0}+H_{0}^{1}(\Omega)\right\} \\ D \phi=0 \text { in } z_{u}}} \int_{\Omega} \alpha|D \phi(x)|^{2} d x<+\infty .
$$

Let $Y=(0,1)^{n}$ be the unit cube in $\mathbb{R}^{n}$. By Vitali's covering theorem, there exists a countable family $\left(\Omega_{i}\right)_{i \geq 1}$ of disjoint homothetics, of ratio less than or equal to 1 , of $\Omega$ such that

$$
\left|Y-\bigcup_{i \geq 1} \bar{\Omega}_{i}\right|=0
$$


Let $\left(Z_{u}\right)_{i}$ be the associated family of homothetics of $Z_{u}$. Denote by $Z$ the set

$$
Z=\bigcup_{i \geq 1}\left(Z_{u}\right)_{i}
$$

Let $\chi(x)$ be the characteristic function of the set $Y \backslash Z$. Define the homogenized tensor $A_{\chi}$ associated to the characteristic function $\chi(x)$ by

$$
A_{\chi} \zeta^{t} \cdot \zeta=\inf _{\substack{\varphi \in H_{\#}^{1}(Y) \\ \zeta+D \varphi=0 \text { in } z}} \int_{Y} \alpha|\zeta+D \varphi|^{2} d y,
$$

where $\zeta$ is any vector in $\mathbb{R}^{n}$. Let us prove that $A_{\chi}$ is a bounded matrix in $\mathbb{R}^{n^{2}}$. Let $\phi$ be an admissible test function for (45) with boundary condition $u_{0}(x)=\zeta \cdot x$. In each $\Omega_{i}$ we define a test function $\varphi$ to be the sum of the homothetics of $\phi$ and of $-\zeta \cdot x$. Since $\varphi$ is equal to 0 on the boundary of each $\Omega_{i}$, pasting these contributions together we obtain a function of $H_{\#}^{1}(Y)$. Therefore,

$$
A_{\chi} \zeta^{\boldsymbol{t}} \cdot \zeta<+\infty \quad \forall \zeta \in \mathbb{R}^{n} .
$$

If $\zeta$ is a line $\xi_{i}$ of $\xi$, for $1 \leq i \leq N$, we can obtain a better bound for $A_{\chi} \xi_{i}^{t} \cdot \xi_{i}$. Using $\varphi(x)=-\xi_{i} \cdot x+u_{i}(x)$ as a test function in (46), where $u_{i}$ is the homothetic of the $i^{t h}$ line of $u$, yields

$$
A_{\chi} \xi^{t} \cdot \xi \leq \frac{1}{|\Omega|} \int_{\Omega} \alpha|D u(x)|^{2} d x .
$$

If $u$ is a minimizer for (5), it satisfies

$$
\frac{1}{|\Omega|} \int_{\Omega} \alpha|D u(x)|^{2} d x+\lambda \frac{\left|\Omega \backslash Z_{u}\right|}{|\Omega|}=Q f(\xi) .
$$

Therefore,

$$
A_{\chi} \xi^{t} \cdot \xi+\lambda \frac{\left|\Omega \backslash Z_{u}\right|}{|\Omega|} \leq Q f(\xi)=\min _{0 \leq \theta \leq 1}\left\{\min _{A \in G_{\theta}^{\infty}} A \xi^{t} \cdot \xi+\lambda \theta\right\} .
$$

Since $\int_{Y} \chi(x) d x=\frac{\left|\Omega \backslash Z_{u}\right|}{|\Omega|}$, we deduce that $\theta=\frac{\left|\Omega \backslash Z_{u}\right|}{|\Omega|}$ and $A=A_{\chi}$ realize the minimum in the right hand side of (48). On the other hand, the optimality condition on $A$ in the computation of $Q f(\xi)$ (see (23), (25), and (26)) shows that at least one eigenvalue of $A$ is equal to $+\infty$ because rank $\xi<n$. This is a contradiction with (47). Thus, there is no minimizer $u \in D_{\xi}$ such that $Z_{u}$ is closed. 


\section{EXISTENCE OF MINIMIZERS FOR A KOHN-STRANG TYPE FUNCTIONAL DEFINED ON DIVERGENCE FREE FIELDS}

This section, which parallels Section 2, is devoted to an analysis of possible minimizers for the functional

$$
\int_{\Omega} f(\sigma) d x
$$

where $\Omega$ is a bounded domain of $\mathbb{R}^{n}$, and $\sigma$ is a divergence free field "which is affine" on the boundary of $\Omega$, i.e.,

$$
\sigma \in \Sigma_{\xi}=\left\{\sigma \in L^{2}\left(\Omega ; \mathbb{R}^{n N}\right) \mid \operatorname{div} \sigma=0 \text { in } \Omega \text { and } \sigma \cdot \vec{n}=\xi \cdot \vec{n} \text { on } \partial \Omega\right\},
$$

where $\vec{n}$ denotes the outward unit normal to $\Omega$ at a point of $\partial \Omega$ and $\operatorname{div} \sigma$ is the $N$-vector whose components are $\left\{\sum_{j=1}^{n} \frac{\partial \sigma_{i j}}{\partial x_{j}}\right\}_{1 \leq i \leq N}$. The specific function $f$ under consideration is similar to that introduced in Section 2. It is taken to bc, for $\eta \in \mathbb{R}^{n N}$,

$$
f(\eta)=\inf \left\{\lambda+\beta^{-1}|\eta|^{2}, 0\right\}
$$

with $0<\beta<+\infty$ and $\lambda<+\infty$.

As in Section 2, the function $f(\eta)$ is in truth the limit of the function

$$
f_{\alpha}(\eta)=\inf \left\{\lambda+\beta^{-1}|\eta|^{2}, \alpha^{-1}|\eta|^{2}\right\}
$$

when $\alpha>0$ tends to zero. In contrast to the setting of Section 2 we shall also prove a partial result for the non-degenerate function $f_{\alpha}$.

Once again the functional defined in (49) is not (sequentially) weakly lower semicontinuous over $L^{2}\left(\Omega ; \mathbb{R}^{n N}\right)$ so that minimizers for (49) over $\Sigma_{\xi}$ defined in (50) need not exist. It is widespread belief that the lower semicontinuous envelope of a functional defined on divergence free fields has for integrand the quasi-convexification of the original integrand. In other words, if

$$
Q f(\eta)=\inf _{s \in \Sigma_{\#}}\left\{\int_{Y} f(\eta+s(y)) d y\right\}
$$


with

$$
\begin{gathered}
\Sigma_{\#}=\left\{s \in L^{2}\left(Y ; \mathbb{R}^{n N}\right) \mid \operatorname{div} s=0 \text { in } Y,\right. \\
\left.s \cdot \vec{n} \text { antiperiodic on } \partial Y, \int_{Y} s d y=0\right\}
\end{gathered}
$$

then the functional

$$
\int_{\Omega} Q f(\sigma) d x
$$

admits (a) minimizer(s) over $\Sigma_{\xi}$ and the minimum value of (53) coïncides with the infimum of (49). The equivalence between sequential weak lower semicontinuity and quasiconvexity in the context of divergence free fields has recently been established in [10]. We will not concern ourselves in this section with a complete proof of the proposed form for the lower semicontinuous envelope.

Let us begin with an explicit formula for the quasi-convexification $Q f(\eta)$ of the original function $f(\eta)$.

THEOREM 3.1. - Let $0 \leq \eta_{1} \leq \ldots \leq \eta_{n}$ be the singular values of $\eta$ (i.e., the square roots of the eigenvalues of $\eta^{t} \eta$ ). Define the function $\rho(\eta)$ by

$$
\rho(\eta)=\sqrt{\sum_{i=p+1}^{n} \eta_{i}^{2}+\frac{1}{p-1}\left(\sum_{i=1}^{p} \eta_{i}\right)^{2}} \text { if } \eta_{p+1}>\frac{1}{p-1} \sum_{i=1}^{p} \eta_{i} \geq \eta_{p}
$$

(there exists a unique $p \in\{2, \ldots, n\}$ such that $\eta_{p+1}>\frac{1}{p-1} \sum_{i=1}^{p} \eta_{i} \geq \eta_{p}$ with the notation $\left.\eta_{n+1}=+\infty\right)$. Then

$$
Q f(\eta)= \begin{cases}\frac{1}{\beta}|\eta|^{2}+\lambda & \text { if } \rho(\eta) \geq \sqrt{\lambda \beta}, \\ \frac{1}{\beta}|\eta|^{2}-\frac{1}{\beta} \rho(\eta)^{2}+2 \sqrt{\frac{\lambda}{\beta}} \rho(\eta) & \text { if } \rho(\eta)<\sqrt{\lambda \beta} .\end{cases}
$$

Remark 3.2. - In space dimension 2, formula (54) simplifies in $\rho(\eta)=\eta_{1}+\eta_{2}$ and the function $Q f$ introduced in Section 2 is recovered upon setting $\alpha=\frac{1}{\beta}$.

In space dimension 3, there are two regimes in formula (54)

$$
\rho(\eta)= \begin{cases}\frac{\eta_{1}+\eta_{2}+\eta_{3}}{\sqrt{2}} & \text { if } \eta_{3} \leq \eta_{1}+\eta_{2} \\ \sqrt{\left(\eta_{1}+\eta_{2}\right)^{2}+\eta_{3}^{2}} & \text { if } \eta_{3}>\eta_{1}+\eta_{2}\end{cases}
$$


Let us also remark that, in any space dimension, when rank $(\eta) \leq n-1$, i.e., when $\eta_{1}=0$, one has $\rho(\eta)=|\eta|$. In this case, we deduce that

$$
Q f(\eta)=C f(\eta)= \begin{cases}\frac{1}{\beta}|\eta|^{2}+\lambda & \text { if }|\eta| \geq \sqrt{\lambda \bar{\beta}} \\ 2 \sqrt{\frac{\lambda}{\beta}}|\eta| & \text { if }|\eta| \leq \sqrt{\lambda \beta}\end{cases}
$$

if rank $(\eta) \leq n-1$. This last remark is at the root of the next theorem.

THEOREM 3.3. - A sufficient condition for (49), (51) to have a minimizer over $\Sigma_{\xi}$ is that at least one of the following conditions holds

(i) $\xi=0$

(ii) $\rho(\xi) \geq \sqrt{\lambda \beta}$

(iii) $\operatorname{rank} \xi=n$ and $\xi_{n}<\frac{1}{n-1} \sum_{i=1}^{n} \xi_{i}$

while (49) has no minimizers over $\Sigma_{\xi}$ when

(iv) $1 \leq \operatorname{rank} \xi \leq n-1$ and $|\xi|<\sqrt{\lambda \beta}$.

Remark 3.4. - Theorem 3.3 leaves open the case rank $\xi=n$, $\rho(\xi)<\sqrt{\lambda \beta}$, and $\xi_{n} \geq \frac{1}{n-1} \sum_{i=1}^{n} \xi_{i}$. We conjecture that in such a case there are no minimizers of (49). As in Proposition 2.5 we could have stated a result ruling out "smooth-type" minimizers in this case. In the spirit of Remark 2.12 we point out that in such a case some of the eigenvalues of the homogenized tensor $A$, entering the computation of $Q f$ (see (3.14) below), are zero. We believe it implies that possible minimizers do not depend on the variables of the corresponding eigendirections, a fact that would violate the affine boundary condition.

THEOREM 3.5. - Consider the minimization of the functional

$$
\int_{\Omega} f_{\alpha}(\sigma) d x
$$

over $\Sigma_{\xi}$, defined in (50), with $f_{\alpha}$ defined in (52). If rank $\xi<n$, then (56) has a minimizer over $\Sigma_{\xi}$ if and only if

$$
\left.\sqrt{\frac{(\beta-\alpha)}{\lambda}}|\xi| \notin\right] \alpha, \beta[\text {. }
$$

Proof of Theorem 3.1. - An argument identical to that which led to (16) would demonstrate that, for the function $f_{\alpha}$ defined in (52),

$$
Q f_{\alpha}(\eta)=\inf _{0 \leq \theta \leq 1}\left\{f_{\alpha}^{\star}(\theta, \eta)+\lambda(1-\theta)\right\}
$$


with

$$
f_{\alpha}^{\star}(\theta, \eta)=\inf _{A \in G_{\theta}^{\alpha}}\left\{A^{-1} \eta^{t} \cdot \eta\right\},
$$

and where $G_{\theta}^{\alpha}$ is identical to the set $G_{\theta}^{\beta}$ defined by (15). Note that the superscript has changed since $\alpha$ is now the varying parameter.

Since $f_{\alpha}$ monotonically increases to $f$ as $\alpha$ goes to 0 , an argument identical to that of Lemma 2.7 yields the monotone convergence of $Q f_{\alpha}$ to $Q f$ as $\alpha$ goes to 0 and,

$$
Q f(\eta)=\inf _{0 \leq \theta \leq 1}\left\{f^{\star}(\theta, \eta)+\lambda(1-\theta)\right\}
$$

with

$$
f^{\star}(\theta, \eta)=\inf _{A \in G_{\theta}^{0}}\left\{A^{-1} \eta^{t} \cdot \eta\right\},
$$

and where $G_{\theta}^{0}$ is the algebraic limit of $G_{\theta}^{\alpha}$ as $\alpha$ goes to 0, i.e., the set of symmetric $n \times n$ matrices with eigenvalues $\lambda_{1}, \ldots, \lambda_{n}$ satisfying

$$
\left\{\begin{array}{l}
0 \leq \lambda_{i} \leq(1-\theta) \beta \\
\sum_{i=1}^{n}\left(\beta-\lambda_{i}\right)^{-1} \leq \frac{\theta-1+n}{\theta \beta}
\end{array}\right.
$$

Since $A^{-1} \eta^{t} \cdot \eta=\operatorname{tr}\left(A^{-1} \eta^{t} \eta\right)$ where $\eta^{t} \eta$ is a $n \times n$ matrix, denoting by $0 \leq \eta_{1} \leq \ldots \leq \eta_{n}$ the singular values of $\eta$ and by $0 \leq \lambda_{1} \leq \ldots \leq \lambda_{n}$ the eigenvalues of $A$, a well-known result of von Neumann (see e.g. [mirsky]) states that

$$
\inf _{A \in G_{\theta}^{0}}\left\{A^{-1} \eta^{t} \cdot \eta\right\}=\inf \sum_{i=1}^{n} \frac{\eta_{i}^{2}}{\lambda_{i}}
$$

where the infimum in the right hand side of (62) has to be taken over all values $\left(\lambda_{i}\right)_{1 \leq i \leq n}$ satisfying

$$
\left\{\begin{array}{l}
\lambda_{i} \leq \ldots \leq \lambda_{n} \\
0 \leq \lambda_{i} \leq(1-\theta) \beta \\
\sum_{i=1}^{n}\left(\beta-\lambda_{i}\right)^{-1} \leq \frac{\theta-1+n}{\theta \beta}
\end{array}\right.
$$

To compute this infimum, let us assume for the moment that none of the constraints $0 \leq \lambda_{i} \leq(1-\theta) \beta$ is active and that the only saturated constraint is

$$
\sum_{i=1}^{n}\left(\beta-\lambda_{i}\right)^{-1}=\frac{\theta-1+n}{\theta \beta} .
$$


In this case, a possible minimizer must satisfy the following Euler-Lagrange equation :

$$
\frac{\eta_{i}^{2}}{\lambda_{i}^{2}}=\frac{C^{2}}{\left(\beta-\lambda_{i}\right)^{2}}, \quad 1 \leq i \leq n,
$$

where $C>0$ is a Lagrange multiplier.

An easy calculation yields

$$
\lambda_{i}=\frac{\beta \eta_{i}}{\eta_{i}+\frac{\theta}{1-\theta} \frac{1}{n-1} \sum_{i=1}^{n} \eta_{i}}, \quad 1 \leq i \leq n .
$$

Note that the $\left(\lambda_{i}\right)$ 's are ordered. One must check that $0 \leq \lambda_{i} \leq(1-\theta) \beta$, for all $1 \leq i \leq n$, which is equivalent to

$$
\eta_{n} \leq \frac{1}{n-1} \sum_{i=1}^{n} \eta_{i}
$$

Note that $0<\lambda_{i}<(1-\theta) \beta$, for all $1 \leq i \leq n$, is equivalent to the strict inequality in (65). If (65) is satisfied, the value of the minimum in (62) is

$$
f^{\star}(\theta, \eta)=\frac{1}{\beta}|\eta|^{2}+\frac{\theta}{\beta(1-\theta)(n-1)}\left(\sum_{i=1}^{n} \eta_{i}\right)^{2} .
$$

If (65) is not satisfied, then one of the constraint $0 \leq \lambda_{i} \leq(1-\theta) \beta$ is saturated. Since $\lambda_{i}=0$ can achieve the minimum in (62) only if $\eta_{i}=0$, we consider the case when one of the eigenvalue $\lambda_{i}$ is equal to $(1-\theta) \beta$. Let us assume that $\lambda_{n}=(1-\theta) \beta$ and that all the other eigenvalues satisfy $0<\lambda_{i}<(1-\theta) \beta, 1 \leq i \leq n-1$. The minimization in the right hand side of (62) becomes a $(n-1)$-dimensional problem with the single active constraint

$$
\sum_{i=1}^{n-1}\left(\beta-\lambda_{i}\right)^{-1}=\frac{\theta-2+n}{\theta \beta} .
$$

A computation similar to the previous one yields

$$
\lambda_{i}=\frac{\beta \eta_{i}}{\eta_{i}+\frac{\theta}{1-\theta} \frac{1}{n-2} \sum_{i=1}^{n-1} \eta_{i}}, \quad 1 \leq i \leq n-1 .
$$

One must check again that $0 \leq \lambda_{i} \leq(1-\theta) \beta$, for all $1 \leq i \leq n-1$, which is equivalent to

$$
\eta_{n-1} \leq \frac{1}{n-2} \sum_{i=1}^{n-1} \eta_{i}
$$


If (67) is satisfied.(but not (65)), the value of the infimum in (62) is

$$
f^{\star}(\theta, \eta)=\frac{1}{\beta}|\eta|^{2}+\frac{\theta}{(1-\theta) \beta} \eta_{n}^{2}+\frac{\theta}{(1-\theta) \beta(n-2)}\left(\sum_{i=1}^{n-1} \eta_{i}\right)^{2} .
$$

An easy induction argument shows that the minimum in (62) is

$$
f^{\star}(\theta, \eta)=\frac{1}{\beta}|\eta|^{2}+\frac{\theta}{(1-\theta) \beta} \sum_{i=p+1}^{n} \eta_{i}^{2}+\frac{\theta}{(1-\theta) \beta} \frac{1}{p-1}\left(\sum_{i=1}^{p} \eta_{i}\right)^{2}
$$

if $\eta$ satisfies the following condition, denoted by $\left(H_{p}\right)$,

$$
\eta_{p} \leq \frac{1}{p-1} \sum_{i=1}^{p} \eta_{i}
$$

and does not satisfy all previous conditions $\left(H_{q}\right)$ for $p+1 \leq q \leq n$. It is easily seen that, if $\left(H_{p}\right)$ is not satisfied, all previous conditions $\left(H_{q}\right)$ for $p+1 \leq q \leq n$ are not satisfied either, and that $\left(H_{2}\right)$ is always true. Therefore, with the notation $\eta_{n+1}=+\infty$, then exists a unique $p \in\{2,3, \ldots, n\}$ such that

$$
\eta_{p} \leq \frac{1}{p-1} \sum_{i=1}^{p} \eta_{i}<\eta_{p+1} .
$$

Introducing the function

$$
\rho(\eta)=\sqrt{\sum_{i=p+1}^{n} \eta_{i}^{2}+\frac{1}{p-1}\left(\sum_{i=1}^{p} \eta_{i}\right)^{2}}
$$

with $p$ defined by (68), we finally obtain

$$
f^{\star}(\theta, \eta)=\frac{1}{\beta}|\eta|^{2}+\frac{\theta}{(1-\theta) \beta} \rho(\eta)^{2} .
$$

An easy optimization in $\theta$ leads to the announced formula for $Q f(\eta)$.

Remark 3.6. - In the spirit of Remark 2.9, we emphasize that our computation of the quasiconvex envelope $Q f$ does not use in an essential manner the knowledge of the entire $G$-closure, i.e., the set $G_{\theta}^{0}$. Indeed, the minimum of $\left\{A^{-1} \eta^{t} \cdot \eta\right\}$ over all $A^{\prime}$ 's in $G_{\theta}^{0}$ can be computed without 
the explicit knowledge of $G_{\theta}^{0}$ by using the so-called Hashin-Shtrikman variational principle (see [3] or [2]).

Proof of Theorem 3.3. - For any matrix $\xi$, if $\rho(\xi) \geq \sqrt{\lambda \beta}$, then $f(\xi)=Q f(\xi)$, and $\sigma(x)=\xi$ is a minimizer of (49) over $\Sigma_{\xi}$. Now, let $\xi$ be a matrix of rank $n$ such that

$$
\xi_{n}<\frac{1}{n-1} \sum_{i=1}^{n} \eta_{i} .
$$

Then, the homogenized matrix $A$ which achieves the minimum in the left hand side of (62) has eigenvalues $\left(\lambda_{i}\right)_{1 \leq i \leq n}$ satisfying

$$
0<\lambda_{i}<(1-\theta) \beta, \quad 1 \leq i \leq n .
$$

Since the $\left(\lambda_{i}\right)$ 's do not reach the values 0 and $(1-\theta) \beta$, one can repeat the argument of Section 2 concerning the confocal ellipsoïds construction (the parameters $\left(m_{i}\right)_{1 \leq i \leq N}$ of the ellipsoïds are finite and non zero, see (33)). In the present case, the boundary condition on $\partial B_{\rho^{-}}$is a Neumann one, and the matrix $A(x)$ is

$$
A(x)= \begin{cases}\beta I & \text { in } B_{\rho^{+}} \backslash B_{\rho^{-}}, \\ \sum_{i=1}^{n} \lambda_{i} e_{i} \otimes e_{i} & \text { in } \mathbb{R}^{n} \backslash B_{\rho^{+}}\end{cases}
$$

where $\left(\lambda_{i}\right)$ is the minimizer in the right hand side of (62). Apart from this, the second step of the proof of Theorem 2.3 can be repeated mutatis mutandis to yield the existence of a minimizer of (49) over $\Sigma_{\xi}$.

Finally, consider a matrix $\xi$ of rank less than or equal to $n-1$ and such that $\rho(\xi)=|\xi|<\sqrt{\lambda \beta}$. As noticed in Remark 3.2, $Q f(\xi)=C f(\xi)=2 \sqrt{\frac{\lambda}{\beta}}|\xi|$ for such matrices $\xi$. In such a case, assume that $\sigma(x)$ is a minimizer for (49) over $\Sigma_{\xi}$. Then

$$
Q f(\xi)=\frac{1}{|\Omega|} \int_{\Omega} f(\sigma) d x
$$

Define the set

$$
\Omega_{\beta}=\{x \in \Omega \mid \sigma(x) \neq 0\} .
$$

Then

$$
Q f(\xi)=\frac{1}{|\Omega|} \int_{\Omega_{\beta}} \frac{1}{\beta}|\sigma(x)|^{2}+\lambda \frac{\left|\Omega_{\beta}\right|}{|\Omega|} .
$$


Note that

$$
\begin{gathered}
\int_{\Omega} \sigma_{i j} d x=\sum_{k=1}^{n} \int_{\Omega} D_{k}\left(\sigma_{i k} x_{j}\right) d x=\sum_{k=1}^{n} \int_{\partial \Omega} \xi_{i k} x_{j} n_{k} d H^{n-1} \\
=\sum_{k=1}^{n} \int_{\Omega} D_{k}\left(\xi_{i k} x_{j}\right) d x=|\Omega| \xi_{i j}
\end{gathered}
$$

so that $\frac{1}{|\Omega|} \int_{\Omega} \sigma d x=\xi$.

The mapping $\phi$ defined on $\mathbb{R}^{+} \backslash\{0\} \times \mathbb{R}^{n^{2}}$ by

$$
\phi(t, z)=\frac{|z|^{2}}{t}
$$

is convex, and Jensen's inequality implies, in view of (69) that

$$
\begin{aligned}
Q f(\xi) & \geq \frac{\left|\Omega_{\beta}\right|}{|\Omega|} \phi\left(\beta, \frac{1}{\left|\Omega_{\beta}\right|} \int_{\Omega_{\beta}} \sigma(x) d x\right)+\lambda \frac{\left|\Omega_{\beta}\right|}{|\Omega|} \\
& =\frac{|\Omega|}{\beta\left|\Omega_{\beta}\right|}|\xi|^{2}+\lambda \frac{\left|\Omega_{\beta}\right|}{|\Omega|} .
\end{aligned}
$$

Hence, recalling that, since rank $\xi<n$,

$$
Q f(\xi)=\min _{0 \leq \theta \leq 1}\left\{\frac{1}{(1-\theta) \beta}|\xi|^{2}+\lambda(1-\theta)\right\},
$$

we deduce that

$$
\frac{\left|\Omega_{\beta}\right|}{|\Omega|}=1-\theta_{\min },
$$

and that equality holds in (70), i.e.,

$$
\frac{1}{\left|\Omega_{\beta}\right|} \int_{\Omega_{\beta}} \phi(\beta, \sigma(x)) d x=\phi\left(\beta, \frac{1}{\left|\Omega_{\beta}\right|} \int_{\Omega_{\beta}} \sigma(x) d x\right) .
$$

But, for $t_{0}>0, z_{0} \in \mathbb{R}^{n^{2}}$ and any $(t, z) \in(0,+\infty) \times \mathbb{R}^{n^{2}}$,

$$
\phi(t, z)=\phi\left(t_{0}, z_{0}\right)+D \phi\left(t_{0}, z_{0}\right) \cdot\left(z-z_{0}, t-t_{0}\right)+\frac{1}{t}\left|z-\frac{t}{t_{0}} z_{0}\right|^{2}
$$

so that, upon setting

$$
\begin{cases}z=\sigma(x) & , \quad z_{0}=\xi \\ t=\beta, & t_{0}=\beta\left(1-\theta_{\text {min }}\right),\end{cases}
$$


(71) implies that

$$
\sigma(x)=\frac{1}{1-\theta_{\min }} \xi \text { for a.e. } x \in \Omega_{\beta} .
$$

Then $\tilde{\sigma}(x)=\sigma(x)-\xi$ is a divergence-free field that satisfies

$$
\left\{\begin{array}{l}
\tilde{\sigma}(x) \cdot n=0 \text { on } \partial \Omega, \\
\int_{\Omega} \tilde{\sigma}(x) d x=0, \\
\tilde{\sigma}(x)= \begin{cases}\frac{\theta_{\min }}{1-\theta_{\min }} \xi & \text { on } \Omega_{\beta}, \\
0 & \text { on } \Omega \backslash \Omega_{\beta} .\end{cases}
\end{array}\right.
$$

But there are no such fields other than 0 . Thus, for $\xi \neq 0$ and rank $\xi<n$, there are no minimizers for (49).

Proof of Theorem 3.5. - The proof is very similar to that of Theorem 3.3. We start from the formula (57) for $Q f_{\alpha}(\eta)$,

$$
Q f_{\alpha}(\eta)=\min _{0 \leq \theta \leq 1}\left\{f_{\alpha}^{\star}(\theta, \eta)+\lambda(1-\theta)\right\}
$$

where, denoting by $0 \leq \eta_{1} \leq \ldots \leq \eta_{n}$ the singular values of the matrix $\eta$,

$$
f_{\alpha}^{\star}(\theta, \eta)=\inf _{\lambda_{1} \leq \ldots \leq \lambda_{n}} \sum_{i=1}^{n} \frac{\eta_{i}^{2}}{\lambda_{i}} .
$$

In (72) the infimum has to be taken on the following set :

$$
\left\{\begin{array}{l}
\underline{a}(\theta)=\left(\frac{\theta}{\alpha}+\frac{1-\theta}{\beta}\right)^{-1} \leq \lambda_{i} \leq \theta \alpha+(1-\theta) \beta=\bar{a}(\theta) \\
\sum_{i=1}^{n}\left(\lambda_{i}-\alpha\right)^{-1} \leq(\underline{a}(\theta)-\alpha)^{-1}+(n-1)(\bar{a}(\theta)-\alpha)^{-1} . \\
\sum_{i=1}^{n}\left(\beta-\lambda_{i}\right)^{-1} \leq(\beta-\underline{a}(\theta))^{-1}+(n-1)(\beta-\bar{a}(\theta))^{-1}
\end{array}\right.
$$

If rank $\eta<n$, then $\eta_{1}=0$ and it is easily seen that the minimum in (72) is attained for

$$
\lambda_{1}=\left(\frac{\theta}{\alpha}+\frac{1-\theta}{\beta}\right)^{-1} \quad \text { and } \quad \lambda_{i}=\theta \alpha+(1-\theta) \beta, \quad 2 \leq i \leq n .
$$

(In this case the constraint $\sum_{i=1}^{n}\left(\beta-\lambda_{i}\right)^{-1} \leq(\beta-\underline{a}(\theta))^{-1}+(n-1)(\beta-$ $\bar{a}(\theta))^{-1}$ is exactly satisfied.) Therefore, for rank $\eta<n$,

$$
f_{\alpha}^{\star}(\theta, \eta)=\frac{1}{\theta \alpha+(1-\theta) \beta}|\eta|^{2},
$$

and a simple minimization in $\theta$ yields $Q f_{\alpha}(\eta)=C f_{\alpha}(\eta)$, the convexification of $f_{\alpha}$. From here on, the end of the proof follows that of Theorem 3.3. 


\section{A GENERALIZATION OF THE KOHN-STRANG FUNCTIONAL; PARTIAL RESULTS ON THE POSSIBLE EXISTENCE OF MINIMIZERS}

In this short section the setting is that of Section 2, but a more general (non quadratic) functional is considered. Specifically, for $\eta$ a matrix with lines $\left(\eta_{1}, \ldots, \eta_{N}\right)$ in $\mathbb{R}^{n}$,

$$
f(\eta)=\inf \left\{\lambda+\sum_{i=1}^{N} W_{1}\left(\eta_{i}\right), \sum_{i=1}^{N} W_{2}\left(\eta_{i}\right)\right\}
$$

where $W_{1}$ and $W_{2}$ are convex $C^{1}$-function on $\mathbb{R}^{n}$, positively homogeneous of degree $p(1<p<+\infty)$ (i.e., $\left.W_{i}(\lambda a)=\lambda^{p} W_{i}(a), \lambda \geq 0, i=1,2\right)$; it is also assumed that

$$
W_{i}(a) \neq 0, \quad a \neq 0, \quad i=1,2,
$$

and that, for every $b$ in $\mathbb{R}^{n}$, there exists a constant $\gamma(b)>0$ such that

$$
W_{i}(a) \geq W_{i}(b)+D W_{i}(b) \cdot(a-b)+\gamma(b)|a-b|^{p} .
$$

We are unable, in this latter setting, to prove the exact analogue of Theorem 2.3 (or rather of the generalization of Theorem 2.3 to the nondegenerate case) because we lack an explicit construction of the type performed in the proof of Theorem 2.3 whenever rank $\xi=n$. Our result is the following

THEOREM 4.1. - If rank $\xi=1,(5)$, (73) has a minimizer over $D_{\xi}$ if and only if

$$
\begin{aligned}
& \min _{\theta \in(0,1)}\left[\left(\sum_{i=1}^{N} \mu_{i}^{p}\right)\left(\theta W_{1}^{\star}+(1-\theta) W_{2}^{\star}\right)^{\star}(a)+\lambda \theta\right] \\
& \geq \min \left[\left(\sum_{i=1}^{N} \mu_{i}^{p}\right) W_{1}(a)+\lambda,\left(\sum_{i=1}^{N} \mu_{i}^{p}\right) W_{2}(a)\right],
\end{aligned}
$$

where $\xi=\mu \otimes a, \mu \in \mathbb{R}^{N}, a \in \mathbb{R}^{n}$, and $\star$ denotes the Legendre transformation.

Remark 4.2. - The seemingly non explicit character of Theorem 4.1 can be cured whenever a more explicit form is available for $W_{1}$ and $W_{2}$. For Vol. $15, \mathrm{n}^{\circ} 3-1998$. 
example if $W_{1}(a)=(\alpha / p)|a|^{p}, W_{2}(a)=(\beta / p)|a|^{p}, \alpha<\beta$, then (75) is satisfied if and only if

$$
\left.|\xi|^{p} \notin \lambda p\right] \frac{1}{\beta^{\frac{1}{p-1}}}, \frac{1}{\alpha^{\frac{1}{p-1}}}[.
$$

Proof of Theorem 4.1. - The functional (5) fails, once again, to be (sequentially) weakly lower semicontinuous over $H^{1}\left(\Omega, \mathbb{R}^{N}\right)$. Its lower semicontinuous envelope is given by

$$
\int_{\Omega} Q f(\eta) d x
$$

where $Q f$, the quasiconvexification of $f$, is given by

$$
Q f(\eta)=\inf _{\varphi \in W_{\#}^{1, p}\left(Y ; \mathbf{R}^{N}\right)} \int_{Y} f(\eta+D \varphi) d y .
$$

In (76) $Y$ is the unit cube in $\mathbb{R}^{n}$ and $W_{\#}^{1, p}\left(Y ; \mathbb{R}^{N}\right)$ denotes the subspace of $W^{1, p}\left(Y ; \mathbf{R}^{N}\right)$ of periodic functions. An argument identical to that developed at the onset of Section 2 would lead to

$$
Q f(\eta)=\inf _{0 \leq \theta \leq 1}\{f(\theta, \eta)+\lambda \theta\}
$$

with

$$
f(\theta, \eta)=\inf _{\substack{x \in L^{\infty}(Y ;\{0,1\}) \\ \int_{Y}(x) d y=\theta}}\left\{\sum_{i=1}^{N} W_{\chi}\left(\eta_{i}\right)\right\} .
$$

In (78), $W_{\chi}$ is the homogenized energy associated to $\chi$, i.e.,

$$
W_{\chi}(a)=\inf _{\varphi \in W_{\#}^{1, p}(Y ; \mathbb{R})} \int_{Y}\left(\chi(y) W_{1}+(1-\chi(y)) W_{2}\right)(a+D \varphi(y)) d y
$$

see e.g. [12]).

If rank $\xi=1$, then $\xi=\mu \otimes a, a \in \mathbb{R}^{n}, \mu \in \mathbb{R}^{N}$, and because of the homogeneous character of $W_{i}, i=1,2$, (78) becomes

$$
f(\theta, \xi)=\left(\sum_{i=1}^{N} \mu_{i}^{p}\right) g(\theta, a),
$$

with

$$
g(\theta, a)=\inf _{\substack{x \in L \\ \int_{Y}^{\infty}(Y ;\{y) d y=\theta}}\left\{W_{\chi}(a)\right\} .
$$


A lower bound for $g(\theta, a)$ is easily obtained upon introduction of the dual problem for $W_{\chi}$. Specifically, it is a classical result of the theory of homogenization -and a straightforward consequence of von Neumann's min-max theorem- that

$$
W_{\chi}(a)=\sup _{b \in \mathbb{R}^{n}}\left\{b \cdot a-W_{\chi}^{\star}(b)\right\}
$$

where

$$
W_{\chi}^{\star}(b)=\inf _{s \in \Sigma_{\#}}\left\{\int_{Y}\left(\chi(y) W_{1}^{\star}+(1+\chi(y)) W_{2}^{\star}\right)(b+s(y)) d y\right\} .
$$

In (83), $W_{i}^{\star}, i=1,2$, are the Legendre transforms of $W_{1}$ and $W_{2}$, and $\Sigma_{\#}$ is defined by

$$
\begin{aligned}
& \Sigma_{\#}=\left\{s \in L^{2}\left(Y ; \mathbb{R}^{N}\right) \mid \operatorname{div} s=0 \text { in } Y,\right. \\
&\left.s \cdot \vec{n} \text { antiperiodic on } \partial Y, \int_{Y} s d y=0\right\},
\end{aligned}
$$

Taking $s=0$ as test function in (83) implies, in view of (82), that

$$
g(\theta, a) \geq \sup _{b \in \mathbb{R}^{n}}\left\{b \cdot a-\left(\theta W_{1}^{\star}(b)+(1-\theta) W_{2}^{\star}(b)\right)\right\} .
$$

Actually the inequality in (84) is an equality. This latter result is well known in the field of homogenization although we were enable to locate a complete proof in the available literature. A proof is given in Remark 4.3 bclow for the sake of completeness; as such it can be safely skipped by a trusting reader.

Remark 4.3. - Inequality (84) is actually an equality. Indeed, consider the case of the homogenized energy associated to a characteristic function $\chi(y)$, defined on $Y$ as

$$
\chi(y)=\tilde{\chi}\left(y_{1}\right), \text { with } \tilde{\chi}(t)= \begin{cases}1 & \text { if } 0 \leq t \leq \theta \\ 0 & \text { if } 0<t \leq 1\end{cases}
$$

Let $s \in \mathbb{R}$ and $e_{1}$ be the unit vector in the $y_{1}$-direction. Remark that if $\phi_{1}$ and $\phi_{2}$ are any two vectors of $\mathbb{R}^{n}$, with $\theta \phi_{1}+(1-\theta) \phi_{2}=0$, then the function

$$
\varphi\left(y_{1}\right)= \begin{cases}\phi_{1} y_{1} & \text { if } 0 \leq y_{1} \leq \theta \\ \phi_{2} y_{1}+\left(\phi_{1}-\phi_{2}\right) \theta & \text { if } \theta \leq y_{1} \leq 1\end{cases}
$$


is an admissible test function in (79) specialized to the case at hand. Thus

$$
W_{\chi}\left(s e_{1}\right) \leq I:=\inf _{\substack{\phi_{1}, \phi_{2} \in \boldsymbol{R}^{n} \\ \theta \phi_{1}+(1-\theta) \phi_{2}=0}}\left\{\theta W_{1}\left(s e_{1}+\phi_{1}\right)+(1-\theta) W_{2}\left(s e_{1}+\phi_{2}\right)\right\} .
$$

The infimum $I$ is computed as follows :

$$
\begin{aligned}
I=\inf _{\substack{\phi_{1}, \phi_{2} \\
\theta \phi_{1}+(1-\theta) \phi_{2}=0}}\{ & \left\{\sup _{q_{1}}\left[q_{1} \cdot\left(s e_{1}+\phi_{1}\right)-W_{1}^{\star}\left(q_{1}\right)\right]\right. \\
& \left.+(1-\theta) \sup _{q_{2}}\left[q_{2} \cdot\left(s e_{1}+\phi_{2}\right)-W_{2}^{\star}\left(q_{2}\right)\right]\right\}
\end{aligned}
$$

and, upon application of a finite dimensional min-max theorem,

$$
\begin{gathered}
I=\sup _{q_{1}, q_{2}}\left\{s e_{1} \cdot\left(\theta q_{1}+(1-\theta) q_{2}\right) \quad\left(\theta W_{1}^{\star}\left(q_{1}\right)+(1-\theta) W_{2}^{\star}\left(q_{2}\right)\right)\right. \\
\left.+\inf _{\substack{\phi_{1}, \phi_{2} \\
\theta \phi_{1}+(1-\theta) \phi_{2}=0}}\left\{q_{1} \cdot \theta \phi_{1}+q_{2} \cdot(1-\theta) \phi_{2}\right\}\right\} .
\end{gathered}
$$

But the infimum in $\left(\phi_{1}, \phi_{2}\right)$ is $-\infty$ unless $q_{1}=q_{2}$. Thus

$$
W_{\chi}\left(s e_{1}\right) \leq I=\sup _{q \in \mathbf{R}^{n}}\left\{s e_{1} \cdot q-\left(\theta W_{1}^{\star}(q)+(1-\theta) W_{2}^{\star}(q)\right)\right\} .
$$

But, according to (81), (84),

$$
W_{\chi}\left(s e_{1}\right) \geq I
$$

Thus

$$
W_{\chi}\left(s e_{1}\right)=I
$$

Since we could always choose the $y_{1}$-direction to be in the direction of a given vector $a$, the choice of $a=s e_{1}$ is not restrictive and

$$
g(\theta, a)=\sup _{b \in \mathbb{R}^{n}}\left\{b \cdot a-\left(\theta W_{1}^{\star}(b)+(1-\theta) W_{2}^{\star}(b)\right)\right\} .
$$

Let us resume the proof of Theorem 4.1. In view of (80), (87), if rank $\xi=1, \xi=\mu \otimes a$ and

$$
f(\theta, \xi)=\left(\sum_{i=1}^{N} \mu_{i}^{p}\right)\left(\theta W_{1}^{\star}+(1-\theta) W_{2}^{\star}\right)^{\star}(a),
$$

where ()$^{\star}$ stands once again for the Legendre transform. Assume that (75) does not hold, or equivalently, that the infimum in (77) is attained for 
$\theta_{\min } \neq 0,1$. (Note that $g(\theta, a)$ is a continuous function of $\theta$; see [9], (3.21), (3.22) and Lemma 3.9). Then, if $u$ is a minimizer for (5), (73) in $D_{\xi}$, let

$$
\Omega_{1}=\left\{x \in \Omega \mid \sum_{i=1}^{N} W_{1}\left(D u_{i}(x)\right)+\lambda<\sum_{i=1}^{N} W_{2}\left(D u_{i}(x)\right)\right\}
$$

and

$$
\Omega_{2}=\Omega \backslash \Omega_{1}
$$

Then,

$$
\int_{\Omega} f(D u(x)) d x=\int_{\Omega_{1}} \sum_{i=1}^{N} W_{1}\left(D u_{i}(x)\right) d x+\int_{\Omega_{2}} \sum_{i=1}^{N} W_{2}\left(D u_{i}(x)\right) d x+\lambda\left|\Omega_{1}\right| .
$$

Further, by virtue of Jensen's inequality,

$$
\begin{aligned}
Q f(\xi) & =\frac{1}{|\Omega|} \int_{\Omega} f(D u(x)) d x \\
& \geq \frac{\left|\Omega_{1}\right|}{|\Omega|} \sum_{i=1}^{N} W_{1}\left(\frac{1}{\left|\Omega_{1}\right|} \int_{\Omega_{1}} D u_{i}(x) d x\right) \\
& +\frac{\left|\Omega_{2}\right|}{|\Omega|} \sum_{i=1}^{N} W_{2}\left(\frac{1}{\left|\Omega_{2}\right|} \int_{\Omega_{2}} D u_{i}(x) d x\right)+\lambda \frac{\left|\Omega_{1}\right|}{|\Omega|} .
\end{aligned}
$$

Set

$$
\theta=\frac{\left|\Omega_{1}\right|}{|\Omega|}, \xi_{1}=\frac{1}{\left|\Omega_{1}\right|} \int_{\Omega_{1}} D u(x) d x, \xi_{2}=\frac{1}{\left|\Omega_{2}\right|} \int_{\Omega_{2}} D u(x) d x,
$$

and remark that $\theta \xi_{1}+(1-\theta) \xi_{2}=\xi=\mu \otimes a$. Then (90) becomes

$$
\begin{aligned}
Q f(\xi) \geq & \theta \sum_{i=1}^{N} \sup _{b \in \mathbb{R}^{n}}\left\{b \cdot\left(\xi_{1}\right)_{i}-W_{1}^{\star}(b)\right\} \\
& +(1-\theta) \sum_{i=1}^{N} \sup _{b \in \mathbb{R}^{n}}\left\{b \cdot\left(\xi_{2}\right)_{i}-W_{2}^{\star}(b)\right\}+\lambda \theta \\
\geq & \sum_{i=1}^{N} \sup _{b \in \mathbb{R}^{n}}\left\{b \cdot \xi_{i}-\left(\theta W_{1}^{\star}+(1-\theta) W_{2}^{\star}\right)(b)\right\}+\lambda \theta \\
= & \left(\sum_{i=1}^{N} \mu_{i}^{p}\right) \sup _{b \in \mathbb{R}^{n}}\left\{b \cdot a-\left(\theta W_{1}^{\star}+(1-\theta) W_{2}^{\star}\right)(b)\right\}+\lambda \theta .
\end{aligned}
$$


The homogeneous character of degree $\frac{p}{p-1}$ of $W_{i}^{*}(i=1,2)$ has been used in deriving the last equality of (91). In view of (77), (88), the equality holds in (90) or (91), i.e., upon recalling (89),

$$
\left\{\begin{array}{l}
\theta=\theta_{\min } \\
\frac{1}{\left|\Omega_{1}\right|} \int_{\Omega_{1}} W_{1}\left(D u_{i}(x)\right) d x=W_{1}\left(\left(\xi_{1}\right)_{i}\right) \\
\frac{1}{\left|\Omega_{2}\right|} \int_{\Omega_{2}} W_{2}\left(D u_{i}(x)\right) d x=W_{2}\left(\left(\xi_{2}\right)_{i}\right)
\end{array}\right.
$$

Invoking (74) for the first (and last) time we conclude that

$$
D u(x)=\left\{\begin{array}{lll}
\xi_{1} & , & \text { a.e. on } \Omega_{1}, \\
\xi_{2} & , & \text { a.e. on } \Omega_{2}
\end{array}\right.
$$

which is impossible unless $\xi_{1}=\xi_{2}$ by an argument identical to that used at the end of Step 1 of the proof of Theorem 2.3 in Section 2. But $\xi_{1}=\xi_{2}=\xi$ is not possible because $u=\xi \cdot x$ is not a minimizer since $\theta_{\min } \neq 0,1$. The proof of Theorem 2.3 is complete.

Note added in proof. - In a very recent preprint of Allaire and Lods it is demonstrated that Proposition 2.5 holds true even without the assumed technical condition on the set $Z_{u}$. This confirms our conjecture of Remark 2.12, namely that for a bounded open set $\Omega$ there exists no minimizer for (5) over $D_{\xi}$ when $2 \leq \operatorname{rank} \xi \leq n-1$ and $\sum_{i=1}^{n} \xi_{i}<\frac{\lambda}{\alpha}$. Note also that the existence of a minimizer for (5) when rank $\xi=n$ has also been observed by Grabovsky (Bounds and extremal microstructures for two-component composites: a unified treatment based on the translation method, Proc. Roy. Soc. London Series A 452, pp. 919-944, 1996) in the case of periodic boundary conditions.

\section{REFERENCES}

[1] G. Allaire, E. Bonnetier, G. A. Francfort and F. Jouve, Shape optimization by the homogenization method, Num. Math., Vol. 76, 1997, pp. 27-68.

[2] G. AllatRe and R. K. KoHN, Optimal bounds on the effective behavior of a mixture of two well-ordered elastic materials, Quat. Appl. Math., Vol. 51, 1993, pp. 643-674.

[3] M. Avellaneda, Optimal bounds and microgeometries for elastic two-phase composites, SIAM, J. Appl. Math., Vol. 47, 6, 1987, pp. 1216-1228.

[4] J. M. BALL and R. D. JamES, Fine phase mixtures as minimizers of energy, Arch. Rat. Mech. Anal., Vol. 100, 1, 1987, pp. 13-52.

[5] J. M. BALL and F. MURAT, $W^{1, p}$ quasiconvexity and variational problems for multiple integrals, J. Func. Anal.. Vol. 58, 1984, pp. 225-253. 
[6] B. Dacorogna, Direct Methods in the Calculus of Variations, Springer Verlag, Berlin Heidelberg (1989).

[7] B. Dacorogna and P. Marcellini, Existence of minimizers for non quasiconvex integrals, Arch. Rational Mech. Anal., Vol. 131, 1995, pp. 359-399.

[8] G. Dal MAso and R. V. KoHn, The local character of $G$-closure, to appear.

[9] I. FONSECA and G. FRANCFORT, Relaxation in $B V$ versus quasiconvexification in $W^{1, p}$; a model for the interaction between fracture and damage, Calculus of Variations, Vol. 3 , 4, 1995, pp. 407-446.

[10] I. FONSECA and S. MULLER, A-quasiconvexity: a necessary and sufficient condition for $L^{p}$ weak lower semicontinuity under p.d.e. constraints, to appear.

[11] R. V. KOHN and G. STRANG, Optimal design and relaxation of variational problem I, II, III, Comm. Pure and Appl. Math., Vol. 39, 1986, pp. 353-377.

[12] P. MARCELLINI, Periodic solutions and homogenization of nonlinear variational problems, Ann. Mat. Pura Appl., Vol. 1178, 1978, pp. 139-152.

[13] L. MiRsky, On the trace of a matrix product, Math. Nachr. Vol. 20, 1959, pp. 171-174.

[14] F. Murat, Contre-exemples pour divers problèmes où le contrôle intervient dans les coefficients, Ann. Mat. Pura Appl., Vol. 112, 1977, pp. 49-68.

[15] F. MURAT and L. TARTAR, $H$-convergence, to appear in Topics in the mathematical modeling of composite materials, R. V. Koh, ed., series: Progress in Nonlinear Differential Equations and their Applications, Birkhaüser, Boston (french version: mimeographed notes, séminaire d'Analyse Fonctionnelle et Numérique de l'Université d'Alger, 1978.

[16] F. MURAT and L. TaRTAR, Calcul des variations et Homogénéisation, Les Méthodes de I'Homogénéisation Théorie et Applications en Physique, Coll. Dir. Études et Recherches EDF, Eyrolles, 1985, pp. 319-369.

[17] L. TARTAR, Estimations fines de coefficients homogénéisés, Ennio de Giorgi Colloquium, P. Krée ed., Pitman Research Notes in Math., Vol. 125, 1985, pp. 168-187.

(Manuscript received January 9, 1996.) 